RIMS-1675

Folder complexes and multiflow combinatorial dualities

\author{
By
}

Hiroshi HIRAI

$\underline{\text { July } 2009}$

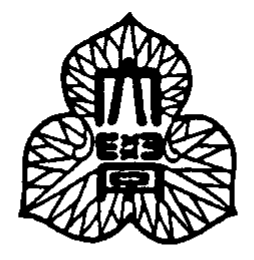

京都大学 数理解析研究所

RESEARCH INSTITUTE FOR MATHEMATICAL SCIENCES

KYOTO UNIVERSITY, Kyoto, Japan 


\title{
Folder complexes and multiflow combinatorial dualities
}

\author{
Hiroshi HIRAI \\ Research Institute for Mathematical Sciences, \\ Kyoto University, Kyoto 606-8502, Japan \\ hirai@kurims.kyoto-u.ac.jp
}

July 2009

\begin{abstract}
In multiflow maximization problems, there are several combinatorial duality relations, such as Ford-Fulkerson's max-flow min-cut theorem for single commodity flows, Hu's max-biflow min-cut theorem for two-commodity flows, Lovász-Cherkassky duality theorem for free multiflows, and so on. In this paper, we provide a unified framework for such multiflow combinatorial dualities by using the notion of a folder complex, which is a certain 2-dimensional polyhedral complex introduced by Chepoi. We show that for a nonnegative weight $\mu$ on terminal set, $\mu$-weighted maximum multiflow problem admits a combinatorial duality relation if and only if $\mu$ is represented by distances among certain subsets in a folder complex, and show that the corresponding combinatorial dual problem is a discrete location problem on the graph of the folder complex. This extends a result of Karzanov for the case of metric weights. Also we give a special optimality criterion to such combinatorial dual problems and give a characterization of such weights in terms of the combinatorial dimension.
\end{abstract}

\section{Introduction}

Let $G$ be an undirected graph with nonnegative edge capacity $c: E G \rightarrow \mathbf{R}_{+}$. Let $S \subseteq V G$ be a set of terminals. A path $P$ in $G$ is called an $S$-path if its ends belong to distinct terminals. A multiflow is a pair $(\mathcal{P}, \lambda)$ of a set $\mathcal{P}$ of $S$-paths and its nonnegative flow-value function $\lambda: \mathcal{P} \rightarrow \mathbf{R}_{+}$satisfying capacity constraint $\sum_{P \in \mathcal{P}: e \in P} \lambda(P) \leq c(e)$ for $e \in E G$. For a nonnegative weight $\mu:\left(\begin{array}{c}S \\ 2\end{array}\right) \rightarrow \mathbf{R}_{+}$on the set of pairs of terminals, the $\mu$-weighted maximum multiflow problem ( $\mu$-problem for short) is formulated as

$$
\begin{array}{cl}
\text { Maximize } & \sum_{P \in \mathcal{P}} \mu\left(s_{P}, t_{P}\right) \lambda(P) \\
\text { subject to } & (\mathcal{P}, \lambda): \text { multiflow for }(G, c ; S),
\end{array}
$$

where $s_{P}, t_{P}$ denote the ends of $P$. The $\mu$-problem (1.1) is a linear problem. So there is a linearly programming duality relation. However, for a special weight $\mu$, the $\mu$-problem admits a combinatorial duality relation. For example, suppose that $S$ is a 2 -set $\{s, t\}$. Then the $\mu$-problem is the maximum flow problem. The max-flow min-cut theorem, due to Ford-Fulkerson [8], says that the maximum flow value is equal to the minimum $s$ - $t$ cut value. Suppose that $S$ is a four-set $\left\{s, t, s^{\prime}, t^{\prime}\right\}$, and $\mu(s, t)=\mu\left(s^{\prime}, t^{\prime}\right)=1$ and other weights are zero. Then the $\mu$-problem is two-commodity flow maximization. $\mathrm{Hu}[14]$ proved that the maximum multiflow value is equal to the minimum of $s s^{\prime}-t t^{\prime}$ mincut value and $s t^{\prime}-t s^{\prime}$ mincut value. Suppose that $\mu$ is all-one weight. Then Lovász [23] and Cherkassky [4] proved that the maximum multiflow value is equal to the half of the sum of $t-S \backslash t$ mincut values over $t \in S$. 
A remarkable result by Karzanov [17] completely characterized metric-weights $\mu$ admitting such a combinatorial duality relation. We shall describe it. Here $\mu$ is called a metric if $\mu$ satisfies the triangle inequality. An undirected graph $\Gamma$ is called a frame if

(i) $\Gamma$ is bipartite,

(ii) $\Gamma$ has no isometric cycle of length greater than four, and

(iii) $\Gamma$ is orientable in the sense that $\Gamma$ has an orientation with the property that, for each 4-cycle $C$, nonadjacent edges have opposite directions with respect to a cyclic ordering of $C$; see the left of Figure 4 .

We denote the shortest path metric of $\Gamma$ by $d_{\Gamma}$.

Theorem $1.1([17])$. Let $\mu$ be a metric on a finite set $S$. Suppose that there exist a frame $\Gamma$ and a map $\phi: S \rightarrow V \Gamma$ such that

$$
\mu(s, t)=d_{\Gamma}(\phi(s), \phi(t)) \quad(s, t \in S) .
$$

Then, for any capacitated graph $(G, c)$ having $S$ as terminal set, the maximum value of the $\mu$-problem (1.1) is equal to the minimum value of the following discrete location problem on $\Gamma$ :

$$
\begin{array}{cl}
\text { Minimize } & \sum_{x y \in E G} c(x y) d_{\Gamma}(\rho(x), \rho(y)) \\
\text { subject to } & \rho: V G \rightarrow V \Gamma, \\
& \rho(s)=\phi(s) \quad(s \in S) .
\end{array}
$$

(Karzanov [17] presented this theorem in the dual form, i.e., the minimum 0-extension problem on a frame is solvable by its metric relaxation, which is the LP-dual to the metric-weighted maximum multiflow problem.) Moreover, if a rational metric $\mu$ cannot be represented by (a dilation of) a submetric of a frame, then there is no such a combinatorial duality relation [18]. From the point of the view in Theorem 1.1, LovászCherkassky duality relation can be understood as follows. Let $\mu$ be the all-one distance on $S$. Then $2 \mu$ is represented as the distance among the leaves of a star consisting of $\# S$ edges. A star is clearly a frame. Then (1.2) coincides with the problem of finding $t-S \backslash t$ mincut for each $s \in S$.

Unfortunately, this theorem cannot be applicable to $\mu$-problems for nonmetric weights $\mu$, such as two-commodity flows. Our previous paper [11] partially extended Theorems 1.1 to a nonmetric version. However this result needs tedious calculations for the tight span and its subdivision, which cause difficulty and inconvenience for a further combinatorial study to $\mu$-problems (1.1). The main contribution (Theorem 2.1) of this paper is a natural extension of Theorem 1.1 for general weights, which provides a useful and flexible framework for multiflow combinatorial dualities. Also this result is an important step of the complete classification of weights $\mu$ having finite fractionality in the next paper [13].

To describe our result, we employee the notion of a folder complex (an F-complex for short) introduced by Chepoi [3, Section 7]. Roughly speaking, an F-complex is a 2-dimensional polyhedral complex obtained by filling a 2-dimensional cell into each 4cycle of a frame. In Section 2, we describe basic definitions and the main result. A key concept that we newly introduce in this paper is the notion of a normal set in an F-complex. Our main result (Theorem 2.1) says that if weight $\mu$ can be realized by the distances among normal sets in an F-complex under $l_{1}$-metrization, then the $\mu$-problem 
has a combinatorial dual problem similar to (1.2). We also give illustrative examples for multiflow combinatorial dualities by F-complexes, including Karzanov-Lomonosov duality relation for anticlique-bipartite commodity flows and seemingly new combinatorial duality relation for a weighted version of two-commodity flows.

Our main result immediately follows from two properties: one is the Helly property of normal sets, and the other one is a decomposition property of finite submetrics on an F-complex. This connection to Helly property sheds a new insight on the multiflow duality. To prove them, in Section 3, we reveal several intriguing properties of shortest paths connecting normal sets in an F-complex, which are previously known for shortest paths connecting vertices of a frame. These properties will also play key roles in the next paper [13]. In Section 4.2, we give a special optimality criterion to combinatorial dual problems, which extends one for the classical tree location problems. Also we verify that weight $\mu$ has such an F-complex realization if and only if $\mu$ has combinatorial dimension most 2. This result has already been suggested by the previous paper [11].

Notation. Let $\mathbf{R}$ and $\mathbf{R}_{+}$be the sets of reals and nonnegative reals, respectively. The set of functions from a set $V$ to $\mathbf{R}$ is denoted by $\mathbf{R}^{V}$. For $p, q \in \mathbf{R}^{V}, p \leq q$ means $p(x) \leq q(x)$ for all $x \in V$.

A function $\mu: S \times S \rightarrow \mathbf{R}$ is called a distance on $S$ if $\mu(s, t)=\mu(t, s) \geq \mu(s, s)=0$ for $s, t \in S$. We regard a nonnegative weight on terminals as a distance. A distance $d$ on $S$ is called a metric if $d$ satisfies the triangle inequality $d(x, z) \leq d(x, y)+d(y, z)$ $(x, y, z \in S)$. For a metric $d$ on $S$, the minimum distance between two subsets $A$ and $B$ is denoted by $d(A, B)$, i.e.,

$$
d(A, B)=\inf _{x \in A, y \in B} d(x, y) .
$$

We simply denote $d(\{x\}, A)$ by $d(x, A)$. For a subset $A \subseteq S$ and a nonnegative real $r$, the ball $B(A, r)$ around $A$ of radius $r$ is defined as

$$
B(A, r)=\{x \in S \mid d(A, x) \leq r\} .
$$

For an undirected graph $G$, the vertex set and the edge set are denoted by $V G$ and $E G$, respectively. An edge joining $x, y \in V G$ is simply denoted by $x y$ or $y x$. Let $d_{G}$ denote the shortest path metric on $V G$ with some specified edge-length. If no edge length is specified, then $d_{G}$ means the shortest path metric by unit edge-length. A subgraph $G^{\prime}$ of $G$ is called isometric if $d_{G^{\prime}}(x, y)=d_{G}(x, y)$ for $x, y \in V G^{\prime}$.

Let $\mathcal{F} \subseteq 2^{S}$ be a set of subsets. We say " $\mathcal{F}$ has the Helly property" if every subset $\mathcal{F}^{\prime} \subseteq \mathcal{F}$ having pairwise nonempty intersection

$$
A \cap B \neq \emptyset \quad\left(A, B \in \mathcal{F}^{\prime}\right)
$$

has nonempty intersection

$$
\bigcap_{A \in \mathcal{F}^{\prime}} A \neq \emptyset \text {. }
$$

A polyhedral complex $\Delta$ is a set of polyhedra in some Euclidean space such that every face of $\sigma \in \Delta$ belongs to $\Delta$, and the nonempty intersection of $\sigma, \sigma^{\prime} \in \Delta$ is a face of both $\sigma$ and $\sigma^{\prime}$. Let $|\Delta|$ denote the underlying set $\bigcup_{\sigma \in \Delta} \sigma$. A member in $\Delta$ is called a cell. A $k$-dimensional cell is simply called a $k$-cell. A 1 -cell is also called an edge. If an edge has ends $p, q$, then it is also denoted by $p q$. A $k$-dimensional polyheral complex is simply called a $k$-complex. For our applications to the multiflow theory, we always assume that $\Delta$ is finite. 


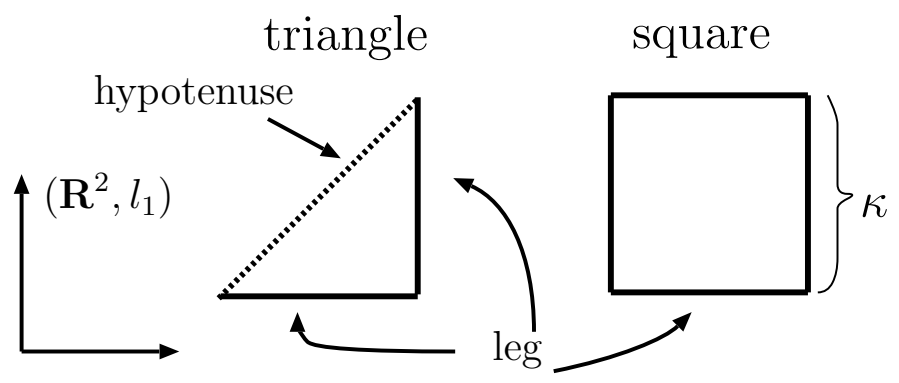

Figure 1: A square and a triangle equipped with the $l_{1}$-distance

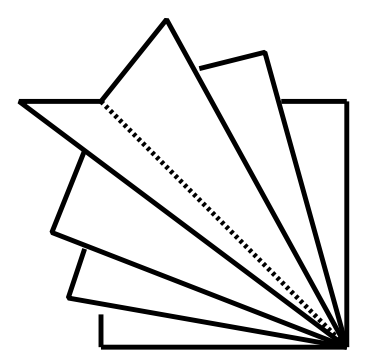

Figure 2: A folder

\section{Definitions, results, and examples}

Let $\Delta$ be a (finite) 2-complex satisfying the following property:

(2.1) (i) For some positive rational $\kappa$, each maximal cell $\sigma$ has an injective continuous map $\varphi_{\sigma}: \sigma \rightarrow \mathbf{R}^{2}$ such that the image $\varphi_{\sigma}(\sigma)$ is

(a) the convex hull of $\{(0,0),(\kappa, 0),(0, \kappa),(\kappa, \kappa)\}$,

(b) the convex hull of $\{(0,0),(\kappa, 0),(\kappa, \kappa)\}$, or

(c) the segment $[(0,0),(\kappa, 0)]$, and

the image of a face of $\sigma$ is a face of $\varphi_{\sigma}(\sigma)$.

(ii) If $\sigma \cap \sigma^{\prime} \neq \emptyset$ for two maximal cells $\sigma, \sigma^{\prime}$, then $\varphi_{\sigma^{\prime}} \circ \varphi_{\sigma}^{-1}$ is an isometry from $\varphi_{\sigma}\left(\sigma \cap \sigma^{\prime}\right)$ to $\varphi_{\sigma^{\prime}}\left(\sigma \cap \sigma^{\prime}\right)$ in the Euclidean distance.

By condition (ii), if 2-cells $\sigma, \sigma^{\prime}$ share a common edge $e$, then $\varphi_{\sigma}(e)=[(0,0),(\kappa, \kappa)]$ implies $\varphi_{\sigma^{\prime}}(e)=[(0,0),(\kappa, \kappa)]$. An edge $e$ of $\Delta$ is called a hypotenuse if $\varphi_{\sigma}(e)=$ $[(0,0),(\kappa, \kappa)]$ for a 2-cell $\sigma$ containing $e$. Other edge is called a leg. In particular, a maximal 1-cell is a leg. $\kappa$ is called the leg-length. A 2-cell $\sigma$ is called a square if its image by $\varphi_{\sigma}$ is (a), and is called a triangle if its image by $\varphi_{\sigma}$ is (b). Namely $\Delta$ is obtained by gluing squares and isosceles right triangles along same type of edges. A folder of $\Delta$ is a square or the union of all triangles sharing one common hypotenuse; see Figure 2.

A more combinatorial and abstract approach is often useful. We can regard $\Delta$ as a pair of a simple graph $\Gamma_{0}$ and a subset $\mathcal{C}$ of its chordless cycles such that 


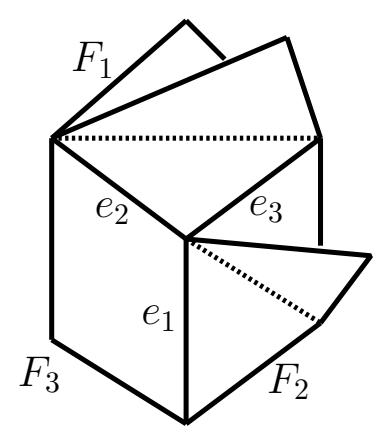

(a)

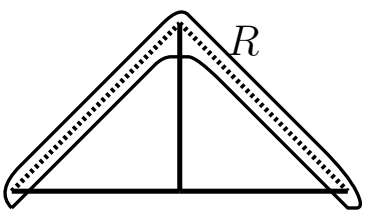

(b)

Figure 3: (a) violation of the flag condition, and (b) violation of the local convexity

(i) the edge set of $\Gamma_{0}$ is partitioned into two types of edges, called legs and hypotenuses, and

(ii) each member of $\mathcal{C}$ is

(a) a 3-cycle (a triangle) consisting of one hypotenuse and two legs, or

(b) a 4-cycle (a square) consisting of four legs,

and every hypotenuse belongs to some triangle.

Indeed, we can take $\Gamma_{0}$ as the 1-skeleton graph of $\Delta$, and take $\mathcal{C}$ as boundary cycles of 2 -cells. Conversely, from $\left(\Gamma_{0} ; \mathcal{C}\right)$ with property $(2.2)$ and a positive rational $\kappa$, we can construct a 2-complex $\Delta$ with property (2.1).

We can endow $|\Delta|$ with the $l_{p}$-metric by the following way. For each cell $\sigma$ and a path $P$ in $\sigma$, the $l_{p}$-length of $P$ is defined by the $l_{p^{-}}$-length of $\varphi_{\sigma^{\prime}}(P)$ in $\left(\mathbf{R}^{2}, l_{p}\right)$ for a maximal cell $\sigma^{\prime}$ containing $\sigma$ (well-defined by (ii)). Then we can define the $l_{p}$-length of a path $P$ in $|\Delta|$ by the sum of the $l_{p}$-length of $P \cap \sigma^{\circ}$ over all cells $\sigma$, where $\sigma^{\circ}$ is the relative interior of $\sigma$. Thus we can define the metric $d_{\Delta, l_{p}}$ on $|\Delta|$ by defining $d_{\Delta, l_{p}}(p, q)$ to be the infimum of the lengths of all paths connecting $p, q$ in $|\Delta|$. In this paper, we are mainly interested in the $l_{1}$-metrization $d_{\Delta, l_{1}}$, which is simply denoted by $d_{\Delta}$.

A simply-connected 2-complex $\Delta$ with property (2.1) is called a folder complex (an F-complex for short) [3, Section 7] if

(i) the intersection of any two folders does not contain incident legs, and

(ii) there are no three folders $F_{i}(i=1,2,3)$ and three distinct legs $e_{i}(i=1,2,3)$ sharing a common vertex such that $e_{i}$ belongs to $F_{j}$ exactly when $i \neq j$.

In fact, this is equivalent to the condition that $|\Delta|$ is a $\operatorname{CAT}(0)$ space under the $l_{2}$ metrization [3, Theorem 7.1]. We particularly call (ii) the flag condition; see Figure 3 (a).

Next we introduce certain subsets of $|\Delta|$. A subset $R$ of $|\Delta|$ is called normal if it satisfies the following property:

(2.4) (i) $R$ coincides with $|\mathcal{K}|$ for a connected subcomplex $\mathcal{K}$ of $\Delta$ with the property that if $\mathcal{K}$ contains a leg $e$, then every cell containing $e$ belongs to $\mathcal{K}$.

(ii) there are no two triangles $\sigma$ and $\sigma^{\prime}$ sharing a leg and a right angle such that $\left(\sigma \cup \sigma^{\prime}\right) \cap R$ coincides with the union of hypotenuses of $\sigma$ and $\sigma^{\prime}$. 

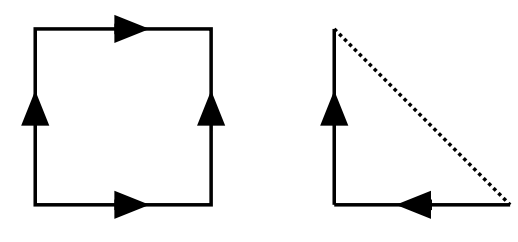

Figure 4: Orientation

We particularly call (ii) the local convexity condition; see Figure 3 (b). Let $\Gamma=\Gamma^{\Delta}$ be the graph consisting of all legs of $\Delta$, called the leg-graph of $\Delta$. Equivalently, $\Gamma$ is the graph obtained by deleting all hypotenuses from the 1-skeleton graph of $\Delta$. The edge-length of $\Gamma$ is defined by $\kappa$ uniformly. An F-complex $\Delta$ is said to be orientable if the leg-graph $\Gamma$ has an orientation such that

(i) in each square, its diagonal edges have same direction. More precisely, orientations of two nonincident edges are opposite each other in a cyclic ordering of the corresponding 4-cycle, and

(ii) in each triangle, its right angle is neither a source nor a sink.

See Figure 4. In fact, the leg-graph of an orientable F-complex is a frame; see Theorem 3.2 in Section 3.1.

For a rational distance $\mu$ on set $S$, a pair $\left(\Delta ;\left\{R_{s}\right\}_{s \in S}\right)$ of an F-complex $\Delta$ and a set $\left\{R_{s}\right\}_{s \in S}$ of normal sets is called an $F$-complex realization of $\mu$ if it satisfies

$$
\mu(s, t)=d_{\Delta}\left(R_{s}, R_{t}\right) \quad(s, t \in S) .
$$

Namely, $\mu$ is realized by the $l_{1}$-distances among normal sets $R_{s}$. Consider the $\mu$-problem (1.1) for a capacitated graph $(G, c)$ with $S \subseteq V G$, and consider the following continuous and discrete location problems on $\Delta$ :

$$
\begin{array}{cl}
\text { Minimize } & \sum_{x y \in E G} c(x y) d_{\Delta}(\rho(x), \rho(y)) \\
\text { subject to } & \rho: V G \rightarrow|\Delta|, \\
& \rho(s) \in R_{s} \quad(s \in S) . \\
\text { Minimize } & \sum c(x y) d_{\Gamma}(\rho(x), \rho(y)) \\
\text { subject to } & \rho: V G \rightarrow V \Gamma, \\
& \rho(s) \in R_{s} \cap V \Gamma \quad(s \in S) .
\end{array}
$$

Now we are ready to describe our main result.

Theorem 2.1. Let $\mu$ be a rational distance on $S$. Suppose that $\mu$ has an F-complex realization $\left(\Delta ;\left\{R_{s}\right\}_{s \in S}\right)$. Then, for any capacitated graph $(G, c)$ with $S \subseteq V G$, the following holds:

(1) The maximum value of the $\mu$-problem (1.1) is equal to the minimum value of the continuous location problem (2.6).

(2) In addition, if $\Delta$ is orientable, then the maximum value of the $\mu$-problem (1.1) is equal to the minimum value of the discrete location problem (2.7). 

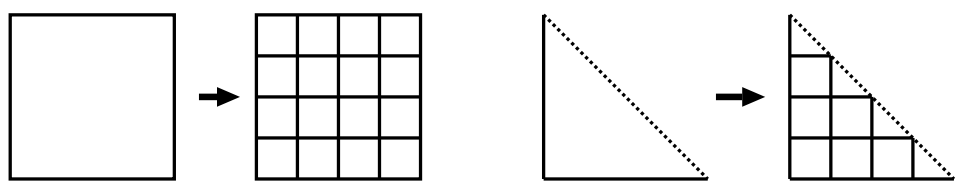

Figure 5: 4-subdivision

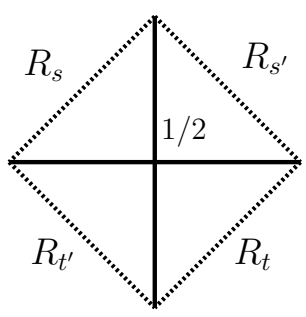

(a)

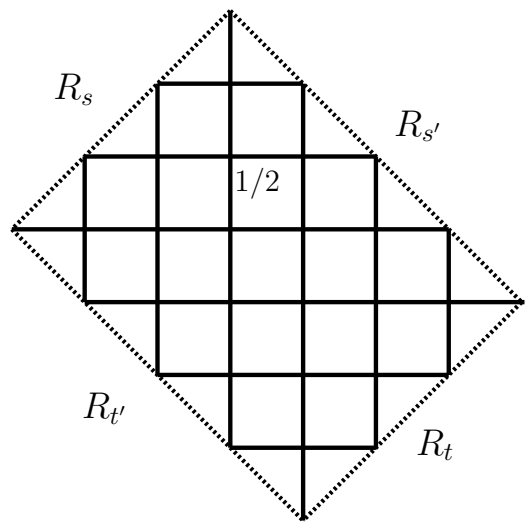

(b)

Figure 6: Two-commodity F-complexes

An F-complex $\Delta$ may be not orientable. By the subdivision operation, we can always obtain an orientable F-complex as follows. For a positive integer $k \geq 2$, the $k$-subdivision $\Delta^{k}$ is obtained from $\Delta$ by subdividing each square into $k^{2}$ squares, subdividing each triangle into $k(k-1) / 2$ squares and $k$ triangles, and subdividing each maximal 1-cell(edge) into $k$ edges as in Figure 5 . The leg-length is set to be $\kappa / k$. One can easily see:

(i) The $k$-subdivision $\Delta^{k}$ is an F-complex for any $k$.

(ii) The 2-subdivision $\Delta^{2}$ is always orientable.

Next we describe illustrative examples of combinatorial duality relations for $\mu$-problems based on Theorem 2.1.

Two-commodity flows. First consider the case where terminal set $S$ is a four-set $\left\{s, t, s^{\prime}, t^{\prime}\right\}$ and distance $\mu$ on $S$ is given as $\mu(s, t)=\mu\left(s^{\prime}, t^{\prime}\right)=1$ and others are zero. Then the $\mu$-problem (1.1) is the two-commodity flow maximization problem [14]. We can realize $\mu$ by the distance on an F-complex as follow. Consider four triangles, glue them around a common right angle, and define the leg-length $\kappa$ to be $1 / 2$, as in Figure 6 (a). The resulting 2-complex is clearly an orientable F-complex. Moreover $\Delta$ realizes $\mu$ as the distance among four hypotenuses, which are normal. Theorem 2.1 (ii) gives a combinatorial duality relation for the two-commodity flow maximization.

Weighted two-commodity flows. Also we can consider a weighted version of twocommodity flows. Let $\mu$ be defined as $\left(\mu(s, t), \mu\left(s^{\prime}, t^{\prime}\right)\right)=(p, q)$ for relatively prime positive integers $p, q$, and other distances are zero. Then consider an F-complex $\Delta$ as in Figure $6(\mathrm{~b})$, which is a subdivision of a rectangle of edge-length ratio $(p: q)$. Let $\kappa=1 / 2$. Clearly $\Delta$ is a F-complex. Again $\mu$ is realized by the distance of the 
four edges of the rectangle, which are normal. Also one can see that $\Delta$ is orientable. Then Theorem 2.1 (ii) yields a combinatorial duality relation, which seems new in the literature.

Anticlique-bipartite commodity flows. A 0-1 distance $\mu$ can be identified with a commodity graph $H$ on $S$ by $s t \in E H \Leftrightarrow \mu(s, t)=1$. We may assume that $H$ has no isolated vertices. The corresponding $\mu$-problem for $(G, c)$ is the maximization of the total sum of flow-values of multiflows connecting pairs of terminals specified by edges of $H$. For a multiflow $f$, the total flow-value with respect to $H$ is denoted by $\|f\|_{H}$. A bipartition $(X, Y)$ of vertex set $V G$ is called a cut, and its cut capacity $c(X, Y)$ is defined to be the sum of the capacity of edges with exactly one end belonging to $X$. A multicut $\mathcal{X}=\left(X_{1}, X_{2}, \ldots, X_{k}\right)(k \geq 2)$ with respect to $H$ is a partition of $V G$ with the property that ends of each edge of $H$ belong to distinct parts of $\mathcal{X}$, and its capacity $c(\mathcal{X})$ is the sum of the capacity of edges whose ends belong to distinct parts of $\mathcal{X}$. For a multiflow $f$ and a multicut $\mathcal{X}$ (w.r.t. $H$ ), the following obvious weak duality relation holds:

$$
\|f\|_{H} \leq c(\mathcal{X}) .
$$

In general, the duality gap is strict (except single- and two-commodity flows). Consider the following relaxation of multicuts. A semi-multicut $\mathcal{X}=\left(X_{1}, X_{2}, \ldots, X_{k}\right)(k \geq 2)$ with respect to $H$ is a set of disjoint subsets of $V G$ with the property that ends of each edge of $H$ belong to distinct sets in $\mathcal{X}$, and its capacity $c(\mathcal{X})$ is defined as

$$
c(\mathcal{X})=\frac{1}{2} \sum_{i=1}^{k} c\left(X_{i}, \overline{X_{i}}\right) .
$$

Again the weak duality (2.9) holds for any multiflow and any semi-multicut, and the duality gap is still strict. However, the class of commodity graphs $H$ admitting strong duality (for semi-multicuts) is not so narrow; recall Lovász-Cherkassky theorem for the case where $H$ is a complete graph. Karzanov and Lomonosov [20] characterized such a class of commodity graphs $H$ as follows.

Theorem $2.2([20])$. Suppose that the set $\mathcal{A}$ of maximal stable sets of commodity graph $H$ has the following properties:

(1) For every triple $A, B, C \in \mathcal{A}$ of maximal stable sets, at least one of $A \cap B, B \cap C$, and $C \cap A$ is empty.

(2) The intersection graph of $\mathcal{A}$ is bipartite.

Then, for any capacitated graph $(G, c)$ having $H$ as commodity graph, the following holds:

$$
\max \left\{\|f\|_{H} \mid \text { multiflow } f\right\}=\min \{c(\mathcal{X}) \mid \text { semi-multicut } \mathcal{X}\} .
$$

See [22], and also see [9] for a polymatroidal approach to this result. A commodity graph with condition (1-2) is called anticlique-bipartite. Here we give an interpretation of this results in terms of an F-complex. Let us construct an F-complex realization for 0 -1 distance $\mu$ corresponding to a commodity graph $H$ with property (1). Let $\Omega_{\mathcal{A}}$ be the intersection graph of $\mathcal{A}$. From $\Omega_{\mathcal{A}}$, we can construct an abstract 2-complex of (2.2). All edges of $\Omega_{\mathcal{A}}$ are supposed be hypotenuses. Add a new vertex $O$, and add a leg $O A$ for each $A \in \mathcal{A}$. Let $\Gamma_{0}$ be the resulting graph. 2-cells $\mathcal{C}$ consists of 3 -cycles of one hypotenuse $A B$ and two legs $O A, O B$ over all intersecting pairs $A, B \in \mathcal{A}$. Define the leg-length $\kappa$ to be $1 / 2$. Let $\Delta$ be the resulting 2-complex, which is the join of $O$ and 


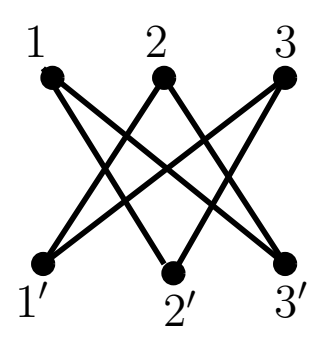

(a)

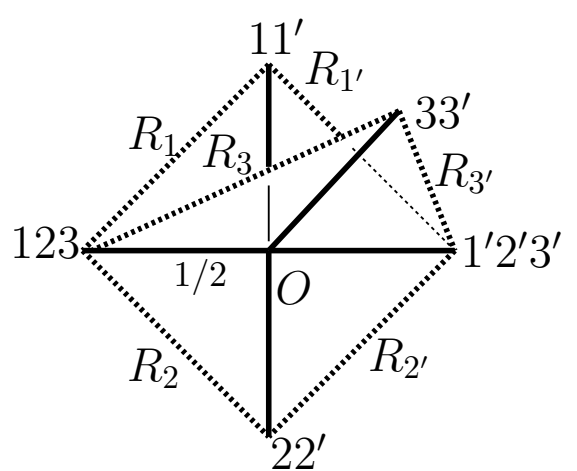

(b)

Figure 7: (a) an anticlique bipartite commodity graph and (b) its F-complex realization
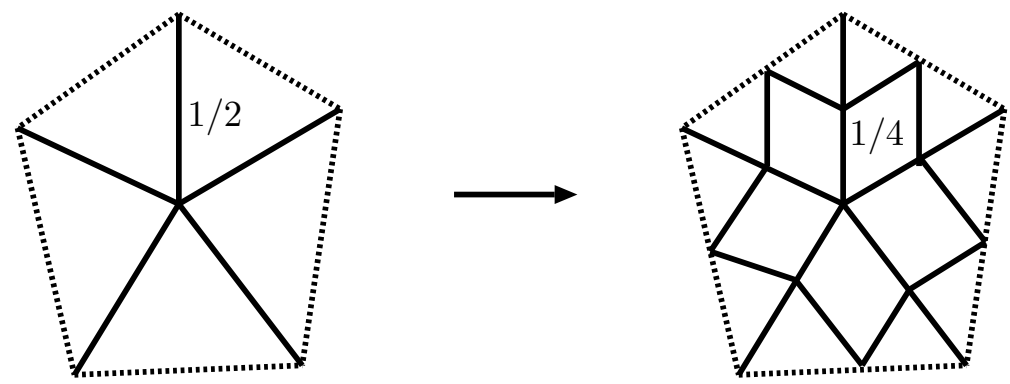

Figure 8: F-complexes for $H=C_{5}$

$\Omega_{\mathcal{A}}$. By the property (1), the girth of $\Omega_{\mathcal{A}}$ is at least four. This fact implies that $\Delta$ is an F-complex; the violation of the flag condition at $O$ implies the existence of a chordless cycle in $\Omega_{\mathcal{A}}$ of length at most three. For $s \in S$, define a normal set $R_{s}$ by

$$
R_{s}= \begin{cases}\text { hypotenuse } A B & \text { if } s \text { belongs to exactly two } A, B \in \mathcal{A}, \\ \operatorname{vertex} A & \text { if } s \text { belongs to exactly one } A \in \mathcal{A} .\end{cases}
$$

Note that every terminal $s$ belongs to at most two maximal stable sets by (1) and the assumption that $H$ has no isolated vertices. Then $\left(\Delta ;\left\{R_{s}\right\}_{s \in S}\right)$ is an F-complex realization. Indeed, $d_{\Delta}\left(R_{s}, R_{t}\right)=0 \Leftrightarrow R_{s} \cap R_{t} \neq \emptyset \Leftrightarrow R_{s} \cap R_{t}=\{A\}$ for $A \in \mathcal{A}$ with $s, t \in A \Leftrightarrow \mu(s, t)=0$, and $R_{s} \cap R_{t}=\emptyset$ implies that $d_{\Delta}\left(R_{s}, R_{t}\right)=1$. Furthermore $\Delta$ is orientable if and only if the condition (2) is fulfilled. Consider the discrete location problem (2.7) for $\left(\Delta ;\left\{R_{s}\right\}_{s \in S}\right)$. For a map $\rho: V G \rightarrow V \Gamma$ feasible to (2.7), the set $\mathcal{X}:=\left(\rho^{-1}(A) \mid A \in \mathcal{A}\right)$ is a semi-multicut, and $\sum_{x y \in E G} c(x y) d_{\Delta}(\rho(x), \rho(y))$ coincides with $c(\mathcal{X})$. Thus Theorem 2.1 implies Karzanov-Lomonosov duality relation above.

Figure 7 illustrates (a) an anticlique-bipartite commodity graph $H$ of $K_{3,3}$ minus one perfect matching, and (b) its F-complex realization $\Delta$. In this case, $\Delta$ is obtained by gluing six triangles of leg-length $1 / 2$, and each $R_{s}$ is a hypotenuse.

Also for nonorientable case, i.e., $H$ violates $(2)$, we obtain a combinatorial duality relation by subdividing $\Delta$ into $\Delta^{2}$, which coincides with the one given in [16]. Figure 8 (a) illustrates an F-complex corresponding to the commodity graph of five-cycle, which is nonorientable, and (b) illustrates its 2-subdivision. See the next paper [13] for further examples. 


\section{Folder complexes and normal sets}

Our main Theorem 2.1 is a consequence of the following two properties of an F-complex $\Delta$; see the paragraph below.

(3.1) (i) For any collection of normal sets $N_{1}, N_{2}, \ldots, N_{k}$ and nonnegative rationals $r_{1}, r_{2}, \ldots, r_{k}$, the collection of balls $\left\{B\left(N_{i}, r_{i}\right)\right\}_{i=1}^{k}$ has the Helly property.

(ii) Suppose that $\Delta$ is orientable. For a map $\rho: X \rightarrow|\Delta|$ on a finite set $X$, define metric $d^{\rho}$ on $X$ by

$$
d^{\rho}(x, y)=d_{\Delta}(\rho(x), \rho(y)) \quad(x, y \in X) .
$$

Then there exist maps $\rho_{i}: X \rightarrow V \Gamma(i \in I)$ such that $d^{\rho}$ is a convex combination of $d^{\rho_{i}}$ and, for any normal set $R, \rho(x) \in R$ implies $\rho_{i}(x) \in R$ $(i \in I)$.

(i) is an extension of [3, Theorem 7.8] for the case where each $N_{i}$ is a singleton set; see Section 4.2. The goal of this section is prove (3.1). In Section 3.1, we introduce some of basic notation. In Section 3.2, we study shortest paths connecting normal sets. By using them, in Section 3.3 we prove (3.1) (i). In Section 3.4, we show a certain decomposition property of shortest paths, and prove (3.1) (ii).

Proof of Theorem 2.1. Here we show Theorem 2.1 by assuming (3.1). The second statement (2) of Theorem 2.1 immediately follows from the first (1) and (3.1) (ii) (and (3.7)). So we show the first statement. Let $\mu$ be a rational distance on finite set $S$. For a capacitated graph $(G, c)$ with terminal set $S$, consider the $\mu$-problem (1.1). As is well-known [22], its LP-dual problem is given by

$$
\begin{aligned}
\text { Minimize } & \sum_{x y \in E G} c(x y) m(x, y) \\
\text { Subject to } & m: \text { metric on } V G, \\
& m(s, t) \geq \mu(s, t) \quad(s, t \in S) .
\end{aligned}
$$

Let $\left(\Delta ;\left\{R_{s}\right\}_{s \in S}\right)$ be an F-complex realization of $\mu$. For a map $\rho$ feasible to (2.6), define metric $d^{\rho}$ on $V G$ as in (3.1) (ii). Then condition $\rho(s) \in R_{s}$ implies

$$
d^{\rho}(s, t)=d_{\Delta}(\rho(s), \rho(t)) \geq d_{\Delta}\left(R_{s}, R_{t}\right)=\mu(s, t),
$$

and thus $d^{\rho}$ is feasible. Hence it suffices to show:

For every rational metric $m$ feasible to (3.2), there exists a map $\rho: V G \rightarrow$ $|\Delta|$ such that $d^{\rho} \leq m$ and $\rho(s) \in R_{s}$ for $s \in S$.

The following argument is based on the idea used in [1, p.421]. Let $m$ be a feasible rational metric. Let $V G=\left\{x_{1}, x_{2}, \ldots, x_{n}\right\}$. Define a map $\rho: V G \rightarrow|\Delta|$ recursively by $\rho\left(x_{k}\right)$ being an arbitrary point in

$$
\bigcap_{s \in S} B\left(R_{s}, m\left(s, x_{k}\right)\right) \cap \bigcap_{i=1}^{k-1} B\left(\rho\left(x_{i}\right), m\left(x_{i}, x_{k}\right)\right) \quad(k=1,2, \ldots, m) .
$$

We show that the set (3.4) is nonempty for all $k$, which implies that $\rho$ is well-defined and satisfies $\rho(s) \in R_{s}$ and $d^{\rho} \leq m$. Indeed, $\rho(s) \in B\left(R_{s}, m(s, s)\right)=R_{s}$, and $\rho\left(x_{j}\right) \in$ $B\left(\rho\left(x_{i}\right), m\left(x_{i}, x_{j}\right)\right)(i<j)$ implies $d_{\Delta}\left(\rho\left(x_{i}\right), \rho\left(x_{j}\right)\right) \leq m\left(x_{i}, x_{j}\right)$. 
We use the induction on $k$. Suppose that (3.4) is nonempty for $i<k$. By rationality, we can take $\rho\left(x_{i}\right)$ as a vertex of $l$-subdivision $\Delta^{l}$ for large $l$. Then each singleton set $\left\{\rho\left(x_{i}\right)\right\}$ is normal in $\Delta^{l}$. Of course, $R_{s}$ is also normal in $\Delta^{l}$. By the Helly property (3.1) (i), it suffices to show that each pairwise intersection is nonemtpy. Note that two balls $B(R, r)$ and $B\left(R^{\prime}, r^{\prime}\right)$ intersect if and only if $d_{\Delta}\left(R, R^{\prime}\right) \leq r+$ $r^{\prime}$. For $s, t \in S$, the nonemtpyness of $B\left(R_{s}, m\left(s, x_{k}\right)\right) \cap B\left(R_{t}, m\left(t, x_{k}\right)\right)$ follows from $m\left(s, x_{k}\right)+m\left(x_{k}, t\right) \geq m(s, t) \geq \mu(s, t)=d_{\Delta}\left(R_{s}, R_{t}\right)$. For $s \in S$ and $i<k$, the nonemtpyness of $B\left(R_{s}, m\left(s, x_{k}\right)\right) \cap B\left(\rho\left(x_{i}\right), m\left(x_{i}, x_{k}\right)\right)$ follows from $m\left(s, x_{k}\right)+m\left(x_{k}, x_{i}\right) \geq$ $m\left(s, x_{i}\right) \geq d_{\Delta}\left(R_{s}, \rho\left(x_{i}\right)\right)$, where the last inequality follows from $\rho\left(x_{i}\right) \in B\left(R_{s}, m\left(s, x_{i}\right)\right)$ for $i<k$ (by induction). For $1 \leq i<j<k$, the nonemtpyness of $B\left(\rho\left(x_{i}\right), m\left(x_{i}, x_{k}\right)\right) \cap$ $B\left(\rho\left(x_{j}\right), m\left(x_{j}, x_{k}\right)\right)$ follows from $m\left(x_{i}, x_{k}\right)+m\left(x_{k}, x_{j}\right) \geq m\left(x_{i}, x_{j}\right) \geq d_{\Delta}\left(\rho\left(x_{i}\right), \rho\left(x_{j}\right)\right)$. Thus we are done.

\subsection{Preliminaries}

Let $\Gamma$ be an undirected graph. The interval $I_{\Gamma}(x, y)$ between $x, y \in V \Gamma$ is defined to be the set $\left\{z \in V G \mid d_{\Gamma}(x, y)=d_{\Gamma}(x, z)+d_{\Gamma}(z, y)\right\}$ (for some specified edge length). An undirected graph $\Gamma$ (with unit edge length) is called modular if for each triple $x, y, z$ of vertices the intersection $I_{\Gamma}(x, y) \cap I_{\Gamma}(y, z) \cap I_{\Gamma}(z, y)$ is nonempty. An element in $I_{\Gamma}(x, y) \cap I_{\Gamma}(y, z) \cap I_{\Gamma}(z, y)$ is called a median of $x, y, z$. A modular graph $\Gamma$ satisfies the so-called quadrangle condition:

For any four vertices $x, u, v, y$ with $d_{\Gamma}(x, u)=d_{\Gamma}(x, v)=1$ and $d_{\Gamma}(u, y)=$ $d_{\Gamma}(v, y)=d_{\Gamma}(x, y)-1$, there exists a common neighbor $w$ of $u$ and $v$ such that $d_{\Gamma}(w, y)=d_{\Gamma}(x, y)-2$.

Indeed, we can take $w$ as a median of $u, v, y$.

A graph is called hereditary modular if all isometric subgraph is modular. Bandelt [2] gave an elegant characterization of hereditary modular graphs as follows.

Theorem 3.1 ([2]). A graph is hereditary modular if and only if it is bipartite and has no isometric cycles of length greater than four.

Therefore a frame is just an orientable hereditary modular graph. Chepoi [3] established a relation between hereditary modular graphs and F-complexes. Here a folder consisting of a single triangle is called a triangle-folder.

Theorem 3.2 ([3, Theorem 7.1]). For a simply-connected 2-complex $\Delta$ satisfying (2.1) without triangle-folders, the following conditions are equivalent:

(i) $\Delta$ is an F-complex.

(ii) its leg-graph $\Gamma$ is a hereditary modular graph without $K_{3,3}$ and $K_{3,3}^{-}$as induced subgraphs.

In addition, (i) $\Rightarrow$ (ii) holds for any simply-connected 2-complex $\Delta$ satisfying (2.1) (possibly including triangle-folders).

$K_{3,3}^{-}$is the graph obtained from $K_{3,3}$ by deleting one edge. Since both $K_{3,3}$ and $K_{3,3}^{-}$ are nonorientable, the leg-graph of an orientable F-complex is a frame. We can partially reconstruct an F-complex $\Delta$ from its leg-graph $\Gamma$ based on the following property:

(i) every 4-cycle of the leg-graph $\Gamma$ belongs to a (unique) folder.

(ii) every 3 -cycle of the 1-skeleton graph of $\Delta$, consisting of two legs and one hypotenuse, is the boundary of a triangle. 
(i) follows from the argument in [3, Section 7]; this is not so obvious, which uses the simply-connectedness, $\mathrm{CAT}(0)$ property of $\Delta$ under the $l_{2}$-metrization, and the GaussBonnet Theorem. (ii) follows from (i). Indeed, the hypotenuse of 3-cycle belongs to some triangle. Use (i) for a possibly appearing 4-cycle of legs.

Therefore, if there is no folder consisting of at most two triangles, then $\Delta$ is completely reconstructed from its leg-graph; there are two ways splitting a square to two triangles. Moreover, for an arbitrary hereditary modular graph without $K_{3,3}$ and $K_{3,3}^{-}$ as induced subgraphs, by filling a folder to each maximal complete bipartite graph $K_{2, n}$ $(n \geq 2)$, we obtain an F-complex without triangle-folders [3].

\subsection{Geodesics}

Here we investigate shortest paths connecting normal sets. Several important properties previously known for shortest paths connecting vertices can be naturally extended for shortest paths connecting normal sets.

Let $\Delta$ be an F-complex. In this subsection, we assume that the leg-length $\kappa$ is equal to 1 for simplicity. We begin with:

$$
\text { For normal sets } N \text { and } M \text {, we have } d_{\Delta}(N, M)=d_{\Gamma}(N \cap V \Gamma, M \cap V \Gamma) \text {. }
$$

Indeed, we can easily modify a (polygonal) geodesic between $N$ and $M$ so that it lies on the union of legs (as in the proof of [11, Proposition 4.2]). For notational simplicity, in the sequel we use the same $d$ for $d_{\Delta}$ and $d_{\Gamma}$, and we denote $I_{\Gamma}(x, y)$ simply by $I(x, y)$. For a normal set $R$, let $\Gamma_{R}$ be the 1 -skeleton graph of the subcomplex of $\Delta$ induced by $R$. The edge length of $e$ of $\Gamma_{R}$ is defined to be 1 if $e$ is a leg and 2 if $e$ is a hypotenuse.

Lemma 3.3. For a normal set $R$, we have $d_{\Gamma_{R}}(x, y)=d(x, y)$ for $x, y \in V \Gamma_{R}$.

Proof. Suppose to the contrary that there are $x, y \in V \Gamma_{R}$ with $d_{\Gamma_{R}}(x, y)>d(x, y)$. Take such $x, y$ with $d_{\Gamma_{R}}(x, y)$ minimum. Then $d_{\Gamma_{R}}(x, y) \geq 4$. Indeed, $d_{\Gamma_{R}}(x, y) \leq 2$ is clearly impossible. Suppose $d_{\Gamma_{R}}(x, y)=3$. Since $\Gamma$ is bipartite, we have $d(x, y)=1$ and therefore $x$ and $y$ are incident in $\Gamma$. Take a shortest path $P$ connecting $x, y$ in $\Gamma_{R}$. $P \cup\{x y\}$ is a 3 -cycle or a 4-cycle in 1-skeleton graph of $\Delta$. In both cases, by (3.6) there is a folder $F$ containing $P \cup\{x y\}$. Then $R$ violates the normality (i) at $F$ by $x y \nsubseteq R$. A contradiction.

Take $z \in I_{\Gamma_{R}}(x, y)$ with $\min \{d(x, z), d(z, y)\} \geq 2$. By minimality assumption, we have $d_{\Gamma_{R}}(x, z)=d(x, z)$ and $d_{\Gamma_{R}}(y, z)=d(y, z)$. Take a median $w$ of $x, y, z$ in $\Gamma$. Then $w \notin R$. Take $w^{\prime} \in I(z, w)$ incident to $z$ (by a leg $z w^{\prime}$ ). We show that there exists a triangle $\sigma$ of vertices $z, w^{\prime}, u$ and hypotenuse $z u$ such that $u \in I_{\Gamma_{R}}(z, x)$ and $\sigma \cap R=z u$. Then there also exists such a triangle $\sigma^{\prime}$ for $I_{\Gamma_{R}}(z, y)$, and $R$ violates the local convexity in $\sigma \cup \sigma^{\prime}$. Take $x^{\prime} \in I_{\Gamma_{R}}(z, x)$ incident to $z$. Suppose that $x^{\prime} z$ is a leg. Then by the quadrangle condition (3.5) for $z, x^{\prime}, w^{\prime}, x$ there exists $x^{\prime \prime}$ incident to $x^{\prime}$ and $w^{\prime}$ with $d(z, x)=d\left(z, x^{\prime \prime}\right)-2$ and therefore there is a folder containing $z, x^{\prime}, x^{\prime \prime}, w^{\prime}$. By the normality (i), $x^{\prime \prime}$ must belong to $R$, and thus $z x^{\prime \prime}$ is a hypotenuse, and there is a required triangle $\sigma$ having $z, w^{\prime}, x^{\prime \prime}$. Suppose that $x^{\prime} z$ is the hypotenuse of some triangle $\tilde{\sigma}$ having vertices $x^{\prime}, z, x_{0}$. By the quadrangle condition for $z, w^{\prime}, x_{0}, x$, there are a common neighbor $x^{\prime \prime}$ of $x_{0}$ and $w^{\prime}$ with $d\left(z, x^{\prime \prime}\right)=d(z, x)-2$, and a folder $F$ containing $z, w^{\prime}, x_{0}, x^{\prime \prime}$. If $x^{\prime \prime}=x^{\prime}$, then $\tilde{\sigma}$ is a required triangle. So $x^{\prime \prime} \neq x^{\prime}$. By the quadrangle condition for $x_{0}, x^{\prime}, x^{\prime \prime}, x$ we can find a folder $F^{\prime}$ violating the flag condition at $x_{0}$ with $\tilde{\sigma}$ and $F$. A contradiction.

We will often use the following bipartite property: 
Lemma 3.4. For a normal set $R$ and a leg $e=x y$, if $e \nsubseteq R$, then we have

$$
d(R, x)-d(R, y) \in\{-1,1\} .
$$

Proof. Let $T$ be the closure of a connected component of $|\Delta| \backslash R$. Since $\Delta$ is simplyconnected, $T \cap R$ is connected. By the normality, $T \cap R$ consists of hypotenuses (or a single point). Therefore $T \cap R \cap V \Gamma$ is contained by one color class of bipartite graph $\Gamma$. The statement immediately follows from this fact.

The next lemma is an extension of the quadrangle condition (3.5). We also call it the quadrangle condition.

Lemma 3.5. For a triple $x, u, v$ of vertices and a normal set $R$, suppose that $d(x, u)=$ $d(x, v)=1$ and $d(u, R)=d(v, R)=d(x, R)-1$.

(1) If $d(x, R)=1$, then there exists a triangle $\sigma$ of vertices $x, u, v$ such that $R \cap \sigma=u v$ (uv is a hypotenuse).

(2) If $d(x, R) \geq 2$, then there exists a common neighbor $w$ of $u$ and $v$ such that $d(w, R)=d(x, R)-2$.

Proof. (1). By Lemma 3.3, $x$ and $y$ are joined by a path $P$ of length 2 in $\Gamma_{R}$. Apply (3.6) for cycle $P \cup\{u x, u v\}$. (2). So suppose that $d(x, R)=k \geq 2$. We use the induction on $k$. Take $u^{\prime}, v^{\prime} \in R$ satisfying $d\left(u, u^{\prime}\right)=d\left(v, v^{\prime}\right)=k-1$ with $d\left(u^{\prime}, v^{\prime}\right)(\leq 2 k)$ minimum. Suppose $d\left(u^{\prime}, v^{\prime}\right)=2 k$. Then $x \in I\left(u^{\prime}, v^{\prime}\right)$. Let $u_{0}^{\prime}$ be a neighbor of $u^{\prime}$ in $I\left(u^{\prime}, x\right)$. By Lemma 3.3, we can take a vertex $u_{1}^{\prime} \in I_{\Gamma_{R}}\left(u^{\prime}, v^{\prime}\right)$ joined to $u^{\prime}$ by a leg or a hypotenuse in $R$. By repeated applications of the quadrangle condition (for $u^{\prime}, u_{0}^{\prime}, \cdot, v^{\prime}$ and for $\left.u_{0}^{\prime}, \cdot, \cdot, v^{\prime}\right)$, as in the last part of the proof of Lemma 3.3, we can find a triangle $\sigma$ of vertices $u^{\prime}, u_{0}^{\prime}, u_{1}^{\prime \prime}$ with $R \cap \sigma=u^{\prime} u_{1}^{\prime \prime}$ and $d\left(v^{\prime}, u_{1}^{\prime \prime}\right)=d\left(v^{\prime}, u^{\prime}\right)-2$. Then $d\left(u_{1}^{\prime \prime}, u\right)=k-1$, and this contradicts to the minimality assumption of $\left(u^{\prime}, v^{\prime}\right)$.

So suppose $d\left(u^{\prime}, v^{\prime}\right)<2 k$. Take a median $w^{*}(\neq x)$ of $u^{\prime}, v^{\prime}, x$ and take a neighbor $w$ of $x$ from $I\left(x, w^{*}\right)$. If $w=v$, then by the quadrangle condition for $x, u, v, u^{\prime}$ we can find a required vertex. Therefore $w \neq u, v$. By the quadrangle condition for $x, u, w, u^{\prime}$, we can find a common neighbor $u^{*}$ of $u, w$ with $d\left(u^{*}, u^{\prime}\right)=d\left(u^{*}, R\right)=k-2$. Similarly we can find a common neighbor $v^{*}$ of $w, v$ with $d\left(v^{*}, v^{\prime}\right)=d\left(v^{*}, R\right)=k-2$. If $u^{*}=v^{*}$, then we are done. So $u^{*} \neq v^{*}$. Let $F_{1}$ and $F_{2}$ be the folders containing $x, u, w, u^{*}$ and $x, v, w, v^{*}$, respectively. Apply the induction to quadrangle $w, u^{*}, v^{*}, R$. Then we can find a folder $F_{3}$ violating the flag condition at $w$ with $F_{1}$ and $F_{2}$. A contradiction.

The next lemma is a certain convexity property of normal sets.

Lemma 3.6. Let $M, N$ be two normal sets. For a vertex $x \in M$, if $d(x, N)>d(M, N)$, then there exists an edge (leg or hypotenuse) $x y \subseteq M$ such that $d(y, N)<d(x, N)$.

Proof. Take a vertex $w$ in $M$ with $d(w, N)=d(M, N)$. Since $M$ is connected, we can take a path $P$ in $\Gamma_{M}$ connecting $x$ and $w$. Among such paths, take $P$ with the following properties:

(a) the integration $I$ of $d(\cdot, N)$ along $P$, i.e.,

$$
I=\sum_{u v: \operatorname{leg} \text { in } P} \frac{d(u, N)+d(v, N)}{2}+\sum_{u v: \text { hypotenuse in } P} d(u, N)+d(v, N)
$$

is minimum. 
(b) $P$ contains legs as much as possible (under property (a)).

Let $P=\left(x=x_{0}, x_{1}, \ldots, x_{m}=w\right)$. Define a sequence $\left\{a_{i}\right\}_{i=0, \ldots, m}$ by $a_{i}=d\left(x_{i}, N\right)$. We show that $\left\{a_{i}\right\}_{i=0, \ldots, m}$ has no triple $a_{i-1}, a_{i}, a_{i+1}$ with the property (i) $a_{i-1}=a_{i}>a_{i+1}$ or (ii) $a_{i-1}<a_{i}>a_{i+1}$. This immediately implies the statement.

Case (i). Suppose to the contrary that $a_{i-1}=a_{i}>a_{i+1}$ holds for some $i$. By the bipartite property, edge $x_{i-1} x_{i}$ is necessarily a hypotenuse. By construction, there is a triangle $\sigma$ of vertices $x_{i-1}, x_{i}, u$ such that $u \notin M$. Also $u \notin N$. Suppose that $x_{i} x_{i+1}$ is a leg. Then by the quadrangle condition for $x_{i}, x_{i+1}, u, N$, there are a common neighbor $v$ of $x_{i+1}, u$ and a folder $F$ containing $x_{i}, x_{i+1}, u, v$. By normality, we have $v \in M$. Then there is a triangle $\sigma^{\prime}$ of vertices $u, v, x_{i}$ in $F$ such that $M$ violates the local convexity at $\sigma \cup \sigma^{\prime}$. A contradiction. Suppose that $x_{i} x_{i+1}$ is a hypotenuse. There is a triangle $\sigma$ of vertices $x_{i}, x_{i+1}, v$ such that $v \notin M$ (by construction). If $v=u$, then $M$ violates the local convexity on $\sigma \cup \sigma^{\prime}$. So $v \neq u$. By the quadrangle condition for $x_{i}, v, u, N$, there is a common neighbor $z\left(\neq x_{i}\right)$ of $v, u$ and a folder $F$ supported by $x_{i}, v, u, z$. Again, by the quadrangle condition for $v, z, x_{i+1}, N$, there is a folder $F^{\prime}$ violating the flag condition at $v$ with $\sigma^{\prime}$ and $F$. A contradiction.

Case (ii). Suppose to the contrary that $a_{i-1}<a_{i}>a_{i+1}$ holds for some $i$. Then at least one of $x_{i-1} x_{i}$ and $x_{i} x_{i+1}$ is a hypotenuse. Otherwise, by the quadrangle condition and the normality, the segment $x_{i-1} x_{i+1}$ belongs to $M$. This contradicts to the construction. Suppose that both $x_{i-1} x_{i}$ and $x_{i} x_{i+1}$ are hypotenuses. Then there are triangles $\sigma, \sigma^{\prime}$ of vertices $x_{i-1}, x_{i}, u$ and $x_{i-1}, x_{i}, v$ (resp.) such that $u, v \notin M$. If $u=v$, then $M$ violates the local convexity on $\sigma \cup \sigma^{\prime}$. Therefore $u \neq v$. Also in this case, we can find a triple of folders violating the flag condition at $u$ (or $v$ ) by repeated applications of the quadrangle condition, as above. Suppose that $x_{i-1} x_{i}$ is a hypotenuse and $x_{i} x_{i+1}$ is a leg. Then there is a triangle $\sigma$ of vertices $x_{i-1}, x_{i}, u$ with $u \notin M$. Again repeated applications of the quadrangle condition imply the existence of a triple of folders violating the flag condition at $u$. A contradiction.

From the proof, we obtain the following:

Lemma 3.7. Let $M, N$ be two normal sets. For two distinct vertices $x, y \in M$ with $d(x, N)=d(y, N)=d(M, N)$, there is a hypotenuse $x z$ in $M$ with $d(z, y)=d(x, y)-2$ and $d(z, N)=d(M, N)$.

Next we discuss the extendability of a path to a shortest path connecting normal sets. Although our goal is Lemma 3.11 below, we derive it from a more general version (Lemma 3.9) for future applications in [13].

An F-complex $\Delta$ is called star-shaped if there is a vertex $p$ such that every folder contains $p$ and no triangle contains $p$ as its right angle. For a vertex $p$ in $\Delta$, let $\Delta_{p}$ be the subcomplex of $\Delta$ consisting of cells containing $p$ and their faces. Clearly $\Delta_{p}$ is also an F-complex. Although $\Delta_{p}$ may not be star-shaped, $\left(\Delta^{2}\right)_{p}$ is always star-shaped.

Lemma 3.8. Let $p$ be a vertex, and let $R$ be a normal set not containing $p$. Suppose that $\Delta_{p}$ is star-shaped. Then a point $x$ in $\left|\Delta_{p}\right|$ at minimum distance from $R$ is a vertex and uniquely determined.

We call this vertex $x$ the gate of $R$ in $\Delta_{p}$; this definition is compatible to that in [7].

Proof. By $p \notin R$ and (2.4) (i), $R$ does not contain a leg in $\Delta_{p}$. Since $\Delta_{p}$ is star-shaped, the boundary (relative to $|\Delta|$ ) consists of legs. By Lemma 3.4, the map $x \mapsto d(x, R)$ on a leg is monotone decreasing or increasing. Hence the minimum attains at a vertex. Suppose that two vertices $x, y$ have the minimum distance from $R$. Consider the case 
where $x$ and $y$ are not incident to $p$ and have a common neighbor $v\left(\in \Delta_{p}\right)$. Then $x, v, p$ and $y, v, p$ belong to folders $F_{1}$ and $F_{2}$, respectively. By the quadrangle condition for $v, x, y, R$, we obtain a folder $F_{3}$ violating the flag condition with $F_{1}$ and $F_{2}$. A contradiction. Suppose other cases. Take a path $P$ connecting $x$ and $y$ in $\Delta_{p}$ passing $p$. By applying the quadrangle condition for three consecutive vertices in $P$ and $R$, we find a vertex $u$ in $\Delta_{p}$ with $d(u, R)<d(x, R)$, or a vertex $z$ in $\Delta_{p}$ with $d(z, R)=d(x, R)$ such that $z$ and $x$ have a common neighbor. The first case is clearly impossible, and the second case reduces to the case above for $(x, z)$.

The extendability of a shortest path passing a vertex $p$ can be locally characterized by the gates in $\Delta_{p}$ (or $\left(\Delta^{2}\right)_{p}$ when $\Delta_{p}$ is not star-shaped).

Lemma 3.9. Let $p$ be a vertex, and let $M, N$ be normal sets not containing $p$. Suppose that $\Delta_{p}$ is star-shaped. Let $x$ and $y$ be the gates of $M$ and $N$ in $\Delta_{p}$, respectively. Then the following two conditions are equivalent:

(1) $d(M, N)=d(M, x)+d(x, p)+d(p, y)+d(y, N)$.

(2) $d(x, y)=d(x, p)+d(p, y)$.

Proof. (1) $\Rightarrow(2)$ is obvious. We show the converse. We first consider the case where $M$ is a singleton $\{w\}$. Our proof is based on that of [3, Lemma 7.6]. Suppose to the contrary that $d(w, N)<d(w, x)+d(x, p)+d(p, y)+d(y, N)$. We take such $\{w\}$ with $k=d(w, x)$ minimum. Note that $d(p, N)=d(p, y)+d(y, N)$ necessarily holds. Suppose the case $k=0(x=w)$. Suppose that $x$ is incident to $p$. Then $d(x, N)=d(p, N)-1$. Take a neighbor $z$ of $p$ from $I(p, y)$. Apply the quadrangle condition for $p, x, z, N$. Then we obtain a common neighbor $u$ of $x, z$ with $d(u, N)=d(z, N)-1$. Since $u$ belongs to $\Delta_{p}$, we have $z \neq y$ and $d(x, y)=3$. Thus $d(u, N)=d(y, N)$ and $u \neq y$, which contradicts to Lemma 3.8. Suppose that $x$ is not incident to $p(d(p, x)=2)$. Let $z$ be a common neighbor of $x$ and $p$. Then $d(z, N)=d(z, p)+d(p, y)+d(y, N)$ by above. Thus $d(x, N)=d(z, N)-1$ holds. Apply the quadrangle condition for $z, x, p, N$. Then we obtain a common neighbor $u$ of $x$ and $p$ with $d(u, N)=d(p, N)-1$. Then $y$ is not incident to $p$, i.e., $d(x, y)=4$. Take a common neighbor $v$ of $p$ and $y$. Apply the quadrangle condition for $p, v, u, N$. Then we obtain a common neighbor $q$ of $v, u$ with $d(q, N)=d(y, N)$. However $q \neq y$ by $d(x, y)=4$. A contradiction to Lemma 3.8.

Suppose $k=1$, i.e., $w$ is incident to $x$. By the assumption and the bipartite property, we have $d(w, N)=d(x, N)-1$. If $x$ is incident to $p$, then by the quadrangle condition for $x, w, p, N$ there is a folder including $w, p, x$, and thus $w$ belongs to $\Delta_{p}$. A contradiction. Suppose that $x$ is not incident to $p$. Take a common neighbor $z$ of $x$ and $p$. Then $z, x, p$ belong to a common folder $F_{1}$. Apply the quadrangle condition for $x, w, z, N$. We obtain a common neighbor $u$ of $w$ and $z$ such that $d(u, N)=d(w, N)-1$. Then $u, w, z, x$ belong to a common folder $F_{2}\left(\neq F_{1}\right)$. Apply the quadrangle condition for $z, u, p, N$. Then we obtain a folder $F_{3}$ violating the flag condition with $F_{1}$ and $F_{2}$. A contradiction.

So suppose $k \geq 2$. Take a neighbor $z$ of $w$ from $I(w, x)$, and take a neighbor $u$ of $z$ from $I(z, x)$. Both gates of $z$ and $u$ in $\Delta_{p}$ are $x$. By minimality assumption, $d(z, N)=d(z, x)+d(x, p)+d(p, y)+d(y, N)$. Therefore $d(w, N)=d(z, N)-1$. By the quadrangle condition for $z, w, u, N$, there is a common neighbor $r$ of $w, u$ such that $d(r, N)=d(z, N)-2$. Also the gate of $r$ in $\Delta_{p}$ is $x$ and $d(r, x)=d(w, x)-1$. Thus $d(r, N)<d(r, x)+d(x, p)+d(p, y)+d(y, N)$. A contradiction to the minimality of $w$.

Next we show that $M$ is general. Suppose that (1) fails for $k=d(M, x)=0(x \in M)$. Suppose that $x$ is incident to $p$. By Lemma 3.6 and the quadrangle condition, we can find a triangle such that its hypotenuse belongs to $M$ and its right angle is $p$. This 
contradicts to the fact that $\Delta_{p}$ is star-shaped. Suppose that $x$ is not incident to $p$. Let $z$ be a common neighbor of $x$ and $p$. Let $F$ be a folder containing $z, x, p$. By Lemma 3.6 and the quadrangle condition, we can find a triangle $\sigma$ of vertices $x, z, u$ such that $x u=M \cap \sigma$ and $d(u, N)=d(x, N)-2$. By the quadrangle condition for $z, p, u, N$, we can find a folder $F^{\prime}$ violating the flag condition with $F$ and $\sigma$. Suppose that (1) fails for $k=d(M, x)>0$. Take $w \in M$ with $d(w, x)=d(M, x)$ such that $d(w, N)$ is minimum. By the singleton case above, $d(w, N)=d(w, x)+d(x, p)+d(p, y)+d(y, N)$ holds, and thus $d(w, N)>d(N, M)$. By Lemma 3.6, there is $z \in M$ incident to $w$ by a leg or a hypotenuse such that $d(z, N)<d(w, N)$. Let $w^{\prime} \in I(w, x)$ be a neighbor of $w$. By repeated applications of the quadrangle condition, we can find a triangle $\sigma$ of vertices $w, w^{\prime}, z^{\prime}$ such that $w^{\prime} z^{\prime}=\sigma \cap M$ and $d\left(z^{\prime}, N\right)=d(w, N)-2$. Consider the distance $d\left(z^{\prime}, x\right)$. Then $d\left(z^{\prime}, x\right)<d(w, x)$ contradicts to the definition of $w$. Therefore $d\left(z^{\prime}, x\right)=d(w, x)=d(M, x)$. However this contradicts to the minimality of $d(w, N)$.

By the same idea of the proof of Lemma 3.8, we have:

Lemma 3.10. For a folder $F$ and a normal set $R$ not meeting the interior of $F$, a point $x$ in $F$ at minimum distance from $R$ is a vertex and uniquely determined.

Also we call $x$ the gate of $R$ in $F$. Since $F=\left|\left(\Delta^{2}\right)_{p}\right|$ for the center vertex $p$ of the 2 -subdivision of $F$, as a corollary of Lemma 3.9, we obtain the following fact, which is an extension of [17, p.87, Claim 3] for the case where $N, M$ are singleton sets.

Lemma 3.11. Let $F$ be a folder, and $M, N$ normal sets not meeting the interior of $F$. Let $x$ and $y$ be the gates of $M$ and $N$ in $F$, respectively. If $x$ and $y$ are not adjacent, then we have

$$
d(M, N)=d(M, x)+2+d(y, N) .
$$

We also give a variant of Lemma 3.11; a proof is similar.

Lemma 3.12. Let $M, N$ be normal sets, and let $\sigma$ be a triangle with vertices $x, y, z$ and hypotenuse yz. Suppose that $M \cap \sigma=y z$ and $x$ is the gate of $N$ in the folder containing $\sigma$. Then we have

$$
d(M, N)=1+d(x, N) .
$$

\subsection{Helly property of normal sets}

The goal of this subsection is to prove:

Theorem 3.13. For an F-complex, the collection of normal sets has the Helly property.

In fact, (3.1) (i) is a simple corollary of it. For a ball $B(N, r)$ for normal set $N$ and rational radius $r$, we can subdivide $\Delta$ into $\Delta^{k}$ and further subdivide $\Delta^{k}$ into $\tilde{\Delta}^{k}$ by splitting squares into two triangles so that $B(N, r)$ is the union of a subcomplex of $\tilde{\Delta}^{k}$.

$$
B(N, r) \text { is normal in } \tilde{\Delta}^{k} \text {. }
$$

Proof. Note that $N$ is also normal in $\tilde{\Delta}^{k}$. Then (2.4) (i) immediately follows from the bipartite property. Suppose to the contrary that there are adjacent two triangles $\sigma, \sigma^{\prime}$ violating (2.4) (ii) with $B(N, r)$. Let $x$ be a common right angle of $\sigma$ and $\sigma^{\prime}$. Then $d(x, N)=r+1 / k$. Let $y$ and $z$ be distinct neighbors of $x$ belonging to $\sigma$ and $\sigma^{\prime}$, respectively. Then $d(y, N)=d(z, N)=r$. By the quadrangle condition for $x, y, z, N$, we can find a folder $F$ violating the flag condition at $x$ with $\sigma$ and $\sigma^{\prime}$. A contradiction. 
We prove Theorem 3.13 by extending Chepoi's shelling argument in [3, p.157]. As Chepoi did, we will remove cells at the maximum distance from some vertex $x$. So we begin with studying the set

$$
M_{x}=\left\{y \in|\Delta| \mid d(y, x)=\max _{u \in|\Delta|} d(u, x)\right\} .
$$

It is easy to see:

$$
M_{x} \text { is the union of isolated vertices and hypotenuses of triangle-folders. }
$$

$M_{x}$ itself may not be normal.

Any normal set $M$ in $M_{x}$ is a vertex or a tree consisting of hypotenuses.

Proof. We can regard $M$ as a graph of hypotenuses. Suppose that $M$ has a cycle $C$ whose vertices are ordered cyclically as $\left(y_{0}, y_{1}, \ldots, y_{m-1}\right)$. Each edge $y_{i} y_{i+1}$ is the hypotenuse of the unique triangle-folder $\sigma_{i}$. Here the indices are taken modulo $m$. Let $y_{i}^{1}$ be the right angle of $\sigma_{i}$. By normality, $y_{i}^{1} \neq y_{i+1}^{1}$ for each $i$. By the quadrangle condition for $y_{i}, y_{i-1}^{1}, y_{i}^{1}, x$, there is a common neighbor $y_{i}^{2}$ of $y_{i-1}^{1}, y_{i}^{1}$ with $d\left(y_{i}^{2}, x\right)=d\left(y_{i}, x\right)-2$ for each $i$. If $y_{i}^{2}=y_{i-1}^{2}$, then we obtain a triple of folders violating the flag condition at $y_{i}^{1}$. Therefore $y_{i}^{2} \neq y_{i-1}^{2}$ for each $i$. Again by the quadrangle condition for $y_{i}^{1}, y_{i}^{2}, y_{i+1}^{2}, x$, there is a common neighbor $y_{i}^{3}$ of $y_{i}^{1}, y_{i+1}^{1}$ with $d\left(y_{i}^{3}, x\right)=d\left(y_{i}^{1}, x\right)-2$ for each $i$. Similarly $y_{i}^{3} \neq y_{i-1}^{3}$ holds for each $i$. Repeat this procedure. Then $d\left(y_{i}^{j}, x\right)$ strictly decreases. In the final step $k=d(x, M)$, we have $y_{i}^{k}=x$ for each $i$. Thus we obtain a triple of folders violating the flag condition at $y_{i}^{k-1}$. A contradiction.

We are ready to prove Theorem 3.13. We use the induction on the size of $\Delta$. Take some vertex $x$. Consider $M_{x}$ and its connected components.

Case 1: $M_{x}$ has a connected component of an isolated vertex $y$. By the construction of $M_{x}$, all neighbors $y_{1}, y_{2}, \ldots, y_{m}$ of $y$ belong to $I_{\Gamma}(y, x)$. There are three cases:

(1) $y$ belongs to a unique maximal 1-cell $y y_{1}(m=1)$.

(2) $y, y_{1}, y_{2}$ are vertices of a triangle $\sigma$ having $y$ as a right angle $(m=2)$.

(3) $y$ belongs to a folder $F$ such that $F$ is a square, or $y$ is one end of the hypotenuse of $F$.

We may assume that $N_{i} \neq\{y\}$ for all $i$. Consider 2-complex $\Delta^{\prime}$ by deleting all cells containing $y$ from $\Delta$. Clearly $\Delta^{\prime}$ is also simply-connected, and thus an F-complex. Let $N_{i}^{\prime}=N_{i} \cap\left|\Delta^{\prime}\right|$. Then each $N_{i}^{\prime}$ is normal in $\Delta^{\prime}$. We show that each pair $N_{i}^{\prime}, N_{j}^{\prime}$ has nonempty intersection on $\left|\Delta^{\prime}\right|$, and then we can apply induction. It suffices to verify it for a pair $i, j$ such that $N_{i}$ and $N_{j}$ have nonempty intersection on the union of cells containing $y$. Suppose (1). Clearly $N_{i} \cap N_{j}$ necessarily contains $y_{1}$, and so does $N_{i}^{\prime} \cap N_{j}^{\prime}$. Suppose (2). By normality, $N_{i} \cap N_{j}$ includes the hypotenuse $y_{1} y_{2}$, and so does $N_{i}^{\prime} \cap N_{j}^{\prime}$. Suppose (3). If $F$ is a square, then $N_{i} \cap N_{j} \cap F=F$, and $N_{i}^{\prime} \cap N_{j}^{\prime}$ contains a vertex $w$ in $F$ diagonal to $y$. If $y$ belongs to the hypotenuse $y w$ of $F$, then $N_{i} \cap N_{j} \cap F$ contains the hypotenuse $y w$, and $N_{i}^{\prime} \cap N_{j}^{\prime}$ contains $w$.

Case 2: $M_{x}$ has no connected component of an isolated vertex. Take a maximal normal set $M \subseteq M_{x}$. Let $y$ be a degree-one vertex of tree $M$, and let $y_{1}$ be a unique vertex incident to $y$ in $M$. Let $\sigma$ be a unique triangle-folder of hypotenuse $y y_{1}$ with right angle $u$. 
If there is another hypotenuse $e$ joined to $y$, then a triangle $\sigma^{\prime}$ having $e$ shares a leg $y u$ with $\sigma$ (in particular $e \subseteq M_{x}$ ).

Proof. Let $e=y y^{\prime}$. Let $\sigma^{\prime}$ be a triangle containing $e$ and let $u^{\prime}$ be the right angle of $\sigma^{\prime}$. By $y \in M_{x}$, we have $d(y, x)-d\left(y^{\prime}, x\right) \in\{0,2\}$. Suppose $d(y, x)=d\left(y^{\prime}, x\right)$. Then $e$ belongs to $M_{x}$, and thus belongs to the unique triangle-folder $\sigma^{\prime}$. Then $y^{\prime} \notin M$ since $y^{\prime} \in M$ implies $e \in M$ by Lemma 3.3. If $u^{\prime} \neq u$, then $M \cup e$ is also normal, and this is a contradiction to the maximality of $M$. Suppose $d(y, x)=d\left(y^{\prime}, x\right)+2$. Suppose $u^{\prime} \neq u$. Apply the quadrangle condition for $y, u, u^{\prime}, x$, and again apply it at $u^{\prime}$. Then we obtain a violating triple of folders. A contradiction. Thus $u^{\prime}=u$.

Consider $\Delta^{\prime}$ by deleting $\sigma$ and $y y_{1}$ from $\Delta$. Clearly $\Delta^{\prime}$ is an F-complex. Suppose that there is no $N_{i}$ with $N_{i} \cap \sigma=y y_{1}$. Let $N_{i}^{\prime}=N_{i} \cap\left|\Delta^{\prime}\right|$ for each $i$. Then each $N_{i}^{\prime}$ is normal and $\left\{N_{i}^{\prime}\right\}$ has pairwise nonempty intersection on $\left|\Delta^{\prime}\right|$. Apply the induction. Therefore we assume that there is $N_{i}$ with $N_{i} \cap \sigma=y y_{1}$. Suppose that there is $N_{j}$ with $N_{i} \cap N_{j}=\{y\}$. Otherwise, consider $\Delta^{\prime}$ as above. Let $N_{k}^{\prime}=N_{k} \backslash\left(y y_{1} \backslash y_{1}\right)$ if $N_{k} \cap \sigma=y y_{1}$, and $N_{k}^{\prime}=N_{k} \cap\left|\Delta^{\prime}\right|$ otherwise. Then each $N_{k}^{\prime}$ with $N_{k} \cap \sigma=y y_{1}$ is connected and thus normal since $y$ is necessarily a degree-one vertex in $\Gamma_{N_{k}}$ from (3.11). Apply the induction. We show that all $N_{k}$ satisfy $N_{i} \cap N_{k} \supseteq\{y\} ; y$ is a desired common point. Suppose that $N_{k}$ contains neither $y y_{1}$ nor $y$. If $y_{1}$ is the gate of $N_{k}$ in $\sigma$, then by Lemma 3.11 we have $0=d\left(N_{j}, N_{k}\right)=d\left(N_{j}, y\right)+2+d\left(y_{1}, N_{k}\right)>0$ (the gate of $N_{j}$ is $y$ ), and this is a contradiction. If $u$ is the gate of $N_{k}$ in $\sigma$, then, by Lemma 3.12, we have $0=d\left(N_{i}, N_{k}\right)=1+d\left(u, N_{k}\right)>0$, and this is a contradiction. So $y$ is the gate of $N_{k}$. By $d\left(y, N_{k}\right)>d\left(N_{i}, N_{k}\right)=0$ and Lemma 3.6, $y$ is joined to a vertex $y^{\prime}$ by a leg or a hypotenuse in $N_{i}$ with $d\left(y, N_{k}\right)>d\left(y^{\prime}, N_{k}\right)$. If $y y^{\prime}$ is a hypotenuse, then $N_{i}$ violates the local convexity by (3.11). A contradiction. If $y y^{\prime}$ is a leg, then by the quadrangle condition for $y, y^{\prime}, u, x$ there is a hypotenuse $e \subseteq N_{i}$ joined to $y$. Again (3.11) leads a contradiction. Thus we complete the proof of Theorem 3.13.

\subsection{Orbits}

We recall the notion of orbits $[17,18]$ with a slight modification by [11]. Edges $e, e^{\prime} \in E \Gamma$ are called mates if there exists a square $\sigma$ in $\Delta$ containing $e, e^{\prime}$ as nonincident edges or there exists a folder $F$ consisting of triangles such that $F$ contains both $e, e^{\prime}$ as legs. Edges $e, e^{\prime}$ are projective if there is a sequence $e=e_{0}, e_{1}, \ldots, e_{m}=e^{\prime}$ such that $e_{i}$ and $e_{i+1}$ are mates. The projectiveness defines an equivalence relation on $E \Gamma$. An equivalence class is called an orbit. The set of all orbits is denoted by $\mathcal{O}=\mathcal{O}^{\Delta}$. The following property is an extension of [18, Statement 2.2].

Proposition 3.14. Let $M, N$ be normal sets. Let $P, Q$ be paths in $\Gamma$ connecting $M$ and $N$. If $P$ is shortest, then $\#(P \cap O) \leq \#(Q \cap O)$ for each orbit $O \in \mathcal{O}$.

Proof. We first consider the case where $M$ is a singleton set $\{x\}$. We use the induction on the length $k$ of $Q$. We may assume that $k \geq 1$ and the length of $P$ is also positive, and assume that vertices $u$ and $v$ following $x$ in $P$ and $Q$ (resp.) are distinct. By the bipartite property, $d(x, N)-d(v, N) \in\{1,-1\}$. Suppose $d(x, N)-d(v, N)=-1$. Then $P \cup\{x v\}$ is a shortest path between $v$ and $N$, and $Q \backslash\{x v\}$ is a path between $v$ and $N$ of length $k-1$. By induction we have $\#(Q \cap O) \geq \#((Q \backslash\{x v\}) \cap O) \geq$ $\#((P \cup\{x v\}) \cap O) \geq \#(P \cap O)$. Suppose $d(x, N)-d(v, N)=1$. Suppose $\# P=1$. By the quadrangle condition, there is a triangle $\sigma$ of vertices $x, u, v$ with $N \cap \sigma=u v$. Therefore $x u$ and $x v$ belong to the same orbit, and $\#(P \cap O) \leq \#(Q \cap O)$ is obvious. Suppose $\# Q \geq \# P \geq 2$. By the quadrangle condition for $x, u, v, N$, we can find a 
common neighbor $w$ of $u, v$ with $d(w, N)=d(x, N)-2$. We can take a shortest path $P^{*}$ connecting $w$ and $N$. Clearly $P^{*} \cup\{u w\}$ is a shortest path connecting $u$ and $N$. By the induction, $\#((P \backslash\{x u\}) \cap O)=\#\left(\left(P^{*} \cup\{u w\}\right) \cap O\right)$. Similarly, \# $((Q \backslash\{x v\}) \cap O) \geq$ $\#\left(\left(P^{*} \cup\{v w\}\right) \cap O\right)$. Since $x u$ and $v w$ are mates and $x v$ and $u w$ are mates, we have $\#(P \cap O)=\#\left(\left(P^{*} \cup\{w u, u x\}\right) \cap O\right)=\#\left(\left(P^{*} \cup\{w v, v x\}\right) \cap O\right) \leq \#(Q \cap O)$.

Suppose that $M$ is not a singleton set. Let $x$ and $y$ be the ends of $P$ and $Q$ belonging to $M$, respectively. By Lemma 3.6 and the singleton case above, it suffices to show the equality $\#(Q \cap O)=\#(P \cap O)$ for the case where $Q$ is also shortest. By above, we may assume $x \neq y$. We use the induction on $d(x, y)$. By Lemma 3.7, we can take $x^{\prime} \in M$ joined to $x$ by a hypotenuse with $d\left(x^{\prime}, N\right)=d(x, N)$ and $d\left(x^{\prime}, y\right)=d(x, y)-2$. There is a triangle of hypotenuse $x x^{\prime}$ and right angle $w$ with $d(w, N)=d(x, N)-1$. Take any shortest path $\tilde{P}$ connecting $x^{\prime}$ and $M$. Then we have $\#(P \cap O)=\#((\tilde{P} \cup\{x w\}) \cap O)=$ $\#\left(\left(\tilde{P} \cup\left\{x^{\prime} w\right\}\right) \cap O\right)=\#(Q \cap O)$, where the last equality follows from the induction.

For a (disjoint) union $U$ of orbits, we can construct a new 2-complex $\Delta^{U}$ from $\Delta$ by identifying the ends of all edges not belonging to $U$. Namely regard $\Delta$ as an abstract 2complex $\left(\Gamma_{0} ; \mathcal{C}\right)$ with property $(2.2)$. Contract all edges not in $U$ in $\Gamma_{0}$ and delete parallel edges and loops (of hypotenuses) appeared, and also delete 1-cycles (loops) and 2-cycles from $\mathcal{C}$. Again the resulting 2-complex $\Delta^{U}$ satisfies (2.1). The leg-graph of $\Delta^{U}$ is denoted by $\Gamma^{U}$. This construction naturally induces the map $(\cdot)^{U}: V \Gamma \rightarrow V \Gamma^{U}$ by defining $x^{U}$ to be the contracted vertex. By extending linearly, we obtain map $(\cdot)^{U}:|\Delta| \rightarrow\left|\Delta^{U}\right|$.

Proposition 3.15. Let $\Delta$ be an orientable F-complex, and let $U$ be the union of some orbits, and let $W=E \Gamma \backslash U$.

(1) $\Delta^{U}$ is an orientable F-complex.

(2) For a normal set $R$ in $\Delta, R^{U}$ is also normal in $\Delta^{U}$.

(3) For a shortest path $P$ in $\Gamma$ connecting normal sets $M$ and $N, P^{U}$ is also a shortest path in $\Gamma^{U}$ connecting $M^{U}$ and $N^{U}$. In particular, we have

$$
d_{\Delta}(M, N)=d_{\Delta^{U}}\left(M^{U}, N^{U}\right)+d_{\Delta^{W}}\left(M^{W}, N^{W}\right) .
$$

Proof. If $\Delta$ has no triangle-folder, then (1) follows from [19, Statement 3.2]. Suppose that $\Delta$ has triangle-folders. Gluing a new triangle to each triangle-folder along the hypotenuse. Then the resulting 2-complex $\Delta^{\prime}$ is also an orientable F-complex without triangle-folders. $U$ is naturally extended to the union $U^{\prime}$ of orbits in $\Delta^{\prime}$. Apply the case above, and delete attached triangles from $\left(\Delta^{\prime}\right)^{U^{\prime}}$.

(3). Suppose that $P^{U}$ is not shortest. Then there is a shorter path $P^{\prime}$ in $\Gamma^{U}$. By adding edges not in $U$ to $P^{\prime}$, we obtain a (not necessarily shortest) path $P^{*}$ in $\Gamma$ connecting $M$ and $N$. Then there is an orbit $O \subseteq U$ such that $\#\left(O \cap P^{*}\right)<\#(O \cap P)$. By Proposition 3.14, $P$ is not shortest. A contradiction.

(2). It suffices to verify the local convexity condition. Suppose that there are a normal set $R$ and two triangles $\sigma_{1}, \sigma_{2}$ such that $R^{U}$ violates the local convexity on the union of (bijective images) $\sigma_{1}^{U}$ and $\sigma_{2}^{U}$. Suppose that $\sigma_{i}$ has vertices $x_{i}, y_{i}, r_{i}$ with hypotenuse $x_{i} y_{i}=R \cap \sigma_{i}(i=1,2)$, and that $\left(r_{1}^{U}, x_{1}^{U}\right)=\left(r_{2}^{U}, x_{2}^{U}\right)$. Consider the gate $g$ of $r_{1}$ in the folder containing $\sigma_{2}$. If $g=r_{2}$, then by Lemma 3.12 we have $d\left(r_{1}, R\right)=$ $d\left(r_{1}, g\right)+1>1=d\left(r_{1}, R\right)$. Therefore $g \neq r_{2}$. Take a shortest path $P$ from $r_{1}$ to $r_{2}$ including $g$, and consider its image $P^{U}$, which is a shortest path connecting $r_{1}^{U}$ and $r_{2}^{U}$ in $\Gamma^{U}$ by (3). However its length is at least 1 . A contradiction to $r_{1}^{U}=r_{2}^{U}$. 

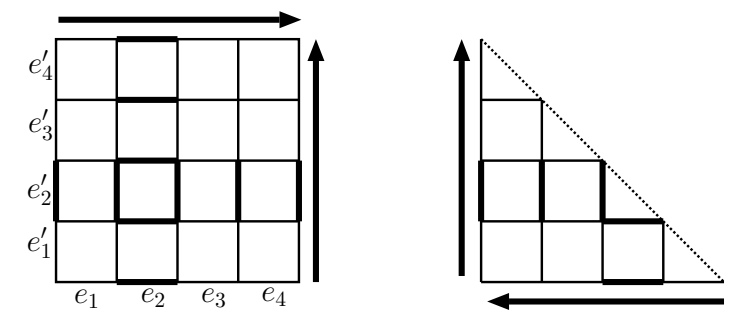

Figure 9: the construction of $U_{i}$; the bold lines represent $U_{2}$

Note that (2) and (3) holds without orientability condition. Now we are ready to prove (3.1) (ii). For a map $\rho: X \rightarrow|\Delta|$, we define metric $d^{\rho}$ on $X$ by

$$
d^{\rho}(x, y)=d_{\Delta}(\rho(x), \rho(y)) \quad(x, y \in X) .
$$

Proposition 3.16. Let $\Delta$ be an orientable F-complex, and let $\Gamma$ be its leg-graph. For any map $\rho: X \rightarrow|\Delta|$ on a finite set $X$, there exist maps $\rho_{i}: X \rightarrow V \Gamma(i \in I)$ such that $d^{\rho}$ is a convex combination of $d^{\rho_{i}}$, and for any normal set $R, \rho(x) \in R$ implies $\rho_{i}(x) \in R$ $(i \in I)$.

We may consider the case where $\rho(X) \subseteq V \Gamma^{k}$ for sufficiently large $k$. Each edge $e$ of $\Gamma$ is subdivided into $k$ edges $e_{1}, e_{2}, \ldots, e_{k}$. Fix an orientation of $\Gamma$ with property (2.5). Suppose that $\left(e_{1}, e_{2}, \ldots, e_{k}\right)$ is arranged from the source to the sink in the orientation of $e$. Let $U_{i}$ be the set of edges projective to $i$-th edge $e_{i}$. By construction, $U_{i} \cap U_{j}=\emptyset$ for $i \neq j$, and $U_{i}$ is the union of orbits of $\Delta^{k}$; see Figure 9. By Proposition $3.15(3)$, we have

$$
d_{\Gamma}(p, q)=\frac{1}{k} \sum_{i=1}^{k} d_{\left(\Gamma^{k}\right)^{U_{i}}}\left(p^{U_{i}}, q^{U_{i}}\right) \quad(p, q \in V \Gamma) .
$$

Each $\Delta^{U_{i}}$ is isomorphic to $\Delta$ up to dilation factor $1 / k$. Therefore $p \mapsto p^{U_{i}}$ induces the map $\phi_{i}:|\Delta| \rightarrow V \Gamma$. Then $\phi_{i} \circ \rho(i=1,2, \ldots, k)$ are desired maps. Also, by construction, $\rho(x) \in \sigma$ implies $\rho_{i}(x) \in \sigma$ for any cell $\sigma \in \Delta$. This implies the latter statement.

\section{Related results}

In this section, we present two results related to our argument. The first one is an optimal criterion of a combinatorial dual problem (2.7), which roughly characterizes the adjacency of extreme points of the feasible region in LP-dual (3.2). This result will play a crucial role in the splitting-off argument [13]. The second one is a characterization of distances of combinatorial dimension at most 2 in terms of F-complex realizations.

\subsection{An optimality criterion for combinatorial dual problems}

Here we show that (2.7) has an optimality criterion of the following type:

If $\rho$ is not optimal, then there exists another $\rho^{\prime}$ close to $\rho$ having the smaller objective value.

We will characterize the closedness in terms of orientations of $\Gamma$. An orientation of $\Gamma$ with property (2.5) is said to be admissible. Admissible orientations are obtained by orienting orbits independently. Such an orientation of an orbit is also said to be 
admissible. Each orbit has exactly two admissible orientations; one is the reverse of the other one. A map $\rho$ feasible to (2.7) is called a potential.

Let $\vec{O}$ be an oriented orbit by an admissible orientation. A potential $\rho^{\prime}$ is called a neighbor of a potential $\rho$ with respect to $\vec{O}$ if for each $x \in V G$ with $\rho^{\prime}(x) \neq \rho(x)$

(i) there is an oriented edge $\overrightarrow{p q} \in \vec{O}$ such that $\rho(x)=p$ and $\rho^{\prime}(x)=q$, or

(ii) there are two oriented edges $\overrightarrow{p q}, \overrightarrow{q r} \in \vec{O}$ belonging to a common 2-cell in $\Delta$ such that $\rho(x)=p$ and $\rho^{\prime}(x)=r$.

Main theorem here is:

Theorem 4.1. If a potential $\rho$ is not optimal, then there exists a neighbor $\rho^{\prime}$ of $\rho$ having the smaller objective value.

Remark 4.2. The combinatorial dual problem (2.7) is a variant of multifacility location problem on graph $\Gamma$ [24]. From the point of the view, Theorem 4.1 can be understood as a generalization of a result of Dearing, Francis, and Lowe [5] for tree location problems (the case where $\Gamma$ is a tree); see also [21, Chapter 3].

The proof is based on the idea used in [12]. A map $\rho$ feasible to (2.6) is called a continuous potential. We denote the distance among points $\rho(x)$ by $d^{\rho}$ as $(3.12)$, and also denote the corresponding objective value $\sum_{x y \in E G} c(x y) d_{\Gamma}(\rho(x), \rho(y))$ simply by $\left\langle c, d^{\rho}\right\rangle$.

Recall the correspondence between metrics $m$ feasible to (3.2) and a continuous potentials $\rho$, discussed in the beginning of Section 3. Namely, for a minimal feasible metric $m$, there is a continuous potential $\rho$ satisfying $m=d^{\rho}$. In general, this correspondence $m \mapsto \rho$ is not one-to-one. However one can establish the one-to-one correspondence by the following trick. Add isolated terminals to $S$ and extend $\mu$ so that $\left\{R_{s}\right\}_{s \in S}$ contains all singleton sets of $V \Gamma$. In this case, the correspondence $m \mapsto \rho$ is one-to-one, and is continuous. Suppose that for a minimal feasible metric $m$ there are two continuous potentials $\rho, \rho^{\prime}$ with $m=d^{\rho}=d^{\rho^{\prime}}$. Suppose $\rho(x) \neq \rho^{\prime}(x)$ for some $x \in V G$. Take minimal cells $\sigma$ and $\sigma^{\prime}$ of $\Delta$ containing $\rho(x)$ and $\rho^{\prime}(x)$, respectively. Suppose that $\sigma$ and $\sigma^{\prime}$ are distinct. Then there is a vertex $v$ in $\sigma$ such that $d_{\Delta}(v, \rho(x)) \neq d_{\Delta}\left(v, \rho^{\prime}(x)\right)$. By the hypothesis, there is a terminal $s$ with $R_{s}=\{v\}$. Then $\rho(s)=\rho^{\prime}(s)=v$ and $d^{\rho}(s, x)=d_{\Gamma}(v, \rho(x)) \neq d_{\Gamma}\left(v, \rho^{\prime}(x)\right)=d^{\rho^{\prime}}(s, x)$. A contradiction. Therefore $\sigma=\sigma^{\prime}$. Since distances $d_{\Delta}(v, \rho(x))\left(=d_{\Delta}\left(v, \rho^{\prime}(x)\right)\right)$ from vertices $v$ of $\sigma$ uniquely determines $\rho(x)$, two maps $\rho$ and $\rho^{\prime}$ must coincide.

We are ready to prove the statement. We remark that if the image of $\rho$ belongs to $V \Gamma$, then $d^{\rho}$ is minimal; this also follows from the fact that $\left\{R_{s}\right\}_{s \in S}$ contains all singleton sets. Suppose that a potential $\rho$ is not optimal. Equivalently, $d^{\rho}$ is not optimal to (3.2). Since $d^{\rho}$ is minimal and nonoptimal, there is another minimal feasible metric $m^{\prime}$ sufficiently close to $d^{\rho}$ such that $\left\langle c, m^{\prime}\right\rangle\left\langle\left\langle c, d^{\rho}\right\rangle\right.$. By the minimality, $m^{\prime}$ is represented by $d^{\rho^{\prime}}$ for a continuous potential $\rho^{\prime}$. Since $m^{\prime}$ is sufficiently close to $d^{\rho}$ and $m^{\prime} \mapsto \rho^{\prime}$ is continuous, we may assume that

$$
d_{\Delta}\left(\rho(x), \rho^{\prime}(x)\right)<\kappa / 2 \quad(x \in V G) .
$$

Recall that $\kappa$ is the leg-length of $\Delta$. Furthermore we may assume that $\rho(V G) \subseteq V \Gamma^{k}$ for sufficiently large $k$. Fix some admissible orientation $\vec{\Gamma}$ of $\Gamma$. Decompose $E \Gamma^{k}$ into $U_{1}, U_{2}, \ldots, U_{k}$ and define maps $\phi_{1}, \phi_{2}, \ldots, \phi_{k}: V \Gamma \rightarrow V \Gamma$ as in the proof of Proposition 3.16. Then we have $d^{\rho^{\prime}}=\sum_{i=1}^{k} d^{\phi_{i} \circ \rho^{\prime}} / k$. Therefore there is an index $i$ such that $\left\langle c, d^{\phi_{i} \circ \rho^{\prime}}\right\rangle\left\langle\left\langle c, d^{\rho}\right\rangle\right.$. We may assume that $1 \leq i \leq k / 2$ by reversing the orientation if 
necessarily. Compare $\phi_{i} \circ \rho^{\prime}$ with $\rho$. By the condition (4.2), if $\left(\phi_{i} \circ \rho^{\prime}\right)(x) \neq \rho(x)$, then

(i) there is an oriented edge $\overrightarrow{p q} \in \overrightarrow{E \Gamma}$ such that $\rho(x)=p$ and $\left(\phi_{i} \circ \rho^{\prime}\right)(x)=q$, or

(ii) there are two oriented edges $\overrightarrow{p q}, \overrightarrow{q r} \in \overrightarrow{E \Gamma}$ belonging to a common 2-cell in $\Delta$ such that $\rho(x)=p$ and $\left(\phi_{i} \circ \rho^{\prime}\right)(x)=r$.

Indeed, we can take a cell $\sigma$ in $\Delta$ containing both $\rho^{\prime}(x)$ and $\rho(x)$. Then $\left(\phi_{i} \circ \rho^{\prime}\right)(x) \neq \rho(x)$ if and only if a shortest path $P$ in $\Gamma^{k}$ connecting $\rho^{\prime}(x)$ and $\rho(x)$ crosses $U_{i}$; recall Figure 9. If $i>k / 2$, then $P$ never crosses $U_{i}$ by (4.2). Thus if $P$ crosses $U_{i}$, then $p=\rho(x)$ is necessarily a source of an oriented leg of $\sigma$. Since $P$ crosses edges in $U_{i}$ at most twice, the position of $\left(\phi_{i} \circ \rho^{\prime}\right)(x)$ is (i) ( $P$ crosses $U_{i}$ once) or (ii) ( $P$ crosses $U_{i}$ twice).

Let $\mathcal{O}=\left\{O_{1}, O_{2}, \ldots, O_{m}\right\}$ be the set of orbits of $\Delta$. As in Section 3.4, we define maps $\varphi_{j}: V \Gamma \rightarrow V \Gamma^{O_{j}}$ by $p \mapsto p^{O_{j}}(j=1,2, \ldots, m)$. By Proposition 3.15 , we have $d^{\rho}=$ $\sum_{j=1}^{k} d^{\varphi_{j} \circ \rho}$ (for any potential $\rho$ ). Therefore we have $\sum_{j=1}^{m}\left\langle c, d^{\varphi_{j} \circ \phi_{i} \circ \rho^{\prime}}\right\rangle<\sum_{j=1}^{m}\left\langle c, d^{\varphi_{j} \circ \rho}\right\rangle$. Then there is an index $j$ with $\left\langle c, d^{\varphi_{j} \circ \phi_{i} \circ \rho^{\prime}}\right\rangle\left\langle\left\langle c, d^{\varphi_{j} \circ \rho}\right\rangle\right.$. Let $\overrightarrow{O_{j}}$ be the admissible orientation of $O_{j}$ induced by $\vec{\Gamma}$. By using $\overrightarrow{O_{j}}$ and $\phi_{i} \circ \rho^{\prime}$, we can construct a required neighbor $\rho^{\prime \prime}$ of $\rho$ with respect to $\overrightarrow{O_{j}}$ as follows. For each $x \in V G$, if there is $\overrightarrow{p q} \in \overrightarrow{O_{j}}$ with $\rho(x)=p$ and $\left(\varphi_{j} \circ \phi_{i} \circ \rho^{\prime}\right)(x)=q^{O_{j}}$ or there are $\overrightarrow{p r}, \overrightarrow{r q} \in \overrightarrow{O_{j}}$ belonging to a common 2-cell with $\rho(x)=p$ and $\left(\varphi_{j} \circ \phi_{i} \circ \rho^{\prime}\right)(x)=q^{O_{j}}$, then define $\rho^{\prime \prime}(x)=q$. For other vertex $x$, define $\rho^{\prime \prime}(x)=\rho(x)$. By construction, $\rho^{\prime \prime}$ is a neighbor of $\rho$ w.r.t. $\overrightarrow{O_{j}}$, and we have

$$
\varphi_{l} \circ \rho^{\prime \prime}=\left\{\begin{array}{ll}
\varphi_{j} \circ \phi_{i} \circ \rho^{\prime} & \text { if } l=j, \\
\varphi_{l} \circ \rho & \text { otherwise, }
\end{array} \quad(l=1,2, \ldots, m) .\right.
$$

Then we have $\left\langle c, d^{\rho^{\prime \prime}}\right\rangle=\sum_{l=1}^{m}\left\langle c, d^{\varphi_{l} \circ \rho^{\prime \prime}}\right\rangle=\sum_{l \neq j}\left\langle c, d^{\varphi_{l} \circ \rho}\right\rangle+\left\langle c, d^{\varphi_{j} \circ \phi_{i} \circ \rho^{\prime}}\right\rangle\left\langle\sum_{l=1}^{m}\left\langle c, d^{\varphi_{l} \circ \rho}\right\rangle=\right.$ $\left\langle c, d^{\rho}\right\rangle$. Thus $\rho^{\prime \prime}$ is a required neighbor.

\subsection{Distances of combinatorial dimension 2}

For a distance $\mu$ on a finite set $S$, define two polyhedral sets $P_{\mu}$ and $T_{\mu}$ in $\mathbf{R}^{S}$ by

$$
\begin{aligned}
& P_{\mu}=\left\{p \in \mathbf{R}^{S} \mid p(s)+p(t) \geq \mu(s, t) \quad(s, t \in S)\right\} \\
& T_{\mu}=\text { the set of minimal elements of } P_{\mu} .
\end{aligned}
$$

$T_{\mu}$ is called the tight span of $\mu[15,6]$. Its dimension $\operatorname{dim} T_{\mu}$ is defined to be the largest dimension of its faces, and is called the combinatorial dimension of $\mu$ [6]. The geometry of $T_{\mu}$ reflects the combinatorial property of $\mu$ as follows.

(i) For a metric $\mu, \operatorname{dim} T_{\mu} \leq 1$ if and only if $\mu$ is a tree metric [6].

(ii) For a distance $\mu, \operatorname{dim} T_{\mu} \leq 1$ if and only if $\mu$ is a distance between subtrees of a tree [10].

(iii) For a rational metric, $\operatorname{dim} T_{\mu} \leq 2$ if and only if $\mu$ is a dilation of a submetric of a frame [18].

The main aim here is to give an extension of (iii) to a nonmetric version as follows.

Theorem 4.3. Let $\mu$ be a rational distance on a finite set $S$. The following two conditions are equivalent: 
(1) $\operatorname{dim} T_{\mu} \leq 2$.

(2) $\mu$ has an F-complex realization.

The larger part of the proof has already been given in [11]. So we sketch it. First we show $(2) \Rightarrow(1)$. Suppose that $\mu$ has an F-complex realization. Then there exists a positive integer $k$ (the denominator of rational leg-length $\kappa$ ) such that LP-dual (3.2) always has a $1 / k$-integral optimal solution. Namely, the dual fractionality of $\mu$ is finite. Then [11, Theorem 1.1] implies $\operatorname{dim} T_{\mu} \leq 2$.

Second, we show $(1) \Rightarrow(2)$. Suppose $\operatorname{dim} T_{\mu} \leq 2$. Then [11] showed that $T_{\mu}$ has a polyhedral subdivision $\Delta$ with property $(2.1)$, and $\left(T_{\mu}, l_{\infty}\right)$ is isometric to $\left(|\Delta|, d_{\Delta}\right)$. For $s \in S$, define a subset $T_{\mu, s}$ by $\left\{p \in T_{\mu} \mid p(s)=0\right\}$. We verify that $\left(\Delta ;\left\{T_{\mu, s}\right\}_{s \in S}\right)$ is an F-complex realization of $\mu$. It is known that $d_{\Delta}\left(T_{\mu, s}, T_{\mu, t}\right)=\mu(s, t)$ [11, Lemma 3.6]. Hence it suffices to verify that $\Delta$ is an F-complex and each $T_{\mu, s}$ is normal. Although one can directly prove that $\Delta$ satisfies (2.3), this approach needs full details of [11, Section 3]. Instead, we use Chepoi's characterization [3] of F-complexes in terms of hyperconvexity. Here a metric space is said to be hyperconvex (in the sense of [1]) if the family of balls (around points) has the Helly property.

Theorem 4.4 ([3, Theorem 7.8]). A locally-finite and simply-connected 2-complex $\Delta$ satisfying (2.1) without triangle-folders is an F-complex if and only if $\left(|\Delta|, d_{\Delta}\right)$ is hyperconvex.

In fact, this theorem holds for any finite and simply-connected 2-complex with property (2.1) possibly having triangle-folders. Indeed, the only if-part follows from Theorem 3.13 (with some care for infinite families and irrational radius). Also the if-part follows from a slight change in Chepoi's proof by the following way. Suppose that $\left(|\Delta|, d_{\Delta}\right)$ is hyperconvex, and suppose to the contrary that there are three folders $F_{1}, F_{2}, F_{3}$ violating (2.3) at a common point $p$. Some of $F_{1}, F_{2}, F_{3}$ may be triangle-folders. In this case, consider 2-subdivision $\Delta^{2}$. Again we can take folders violating (2.3) at $p$ each of which is not a triangle-folder. Then apply the argument in $[3, \mathrm{p} .156]$ to them, which leads a contradiction. So it suffices to verify the following, which is well-known in the literature for metrics $\mu$.

Lemma 4.5. $\left(T_{\mu}, l_{\infty}\right)$ is hyperconvex.

This is an easy consequence of the next lemma.

Lemma 4.6 ([6, p.331, (1.9)]). There exists a map $\phi: P_{\mu} \rightarrow T_{\mu}$ such that

(1) $\|\phi(p)-\phi(q)\|_{\infty} \leq\|p-q\|_{\infty}$ for $p, q \in P_{\mu}$, and

(2) $\phi(p) \leq p$ for $p \in P_{\mu}$, and thus $\phi$ is identical on $T_{\mu}$.

(This lemma holds for nonmetric distances $\mu$ although it was originally stated for metrics.)

The proof of Lemma 4.5. For $\left\{p_{i}\right\}_{i \in I} \subseteq T_{\mu}$ and $\left\{r_{i}\right\}_{i \in I} \subseteq \mathbf{R}_{+}$, suppose that the collection of balls $\left\{B\left(p_{i}, r_{i}\right)\right\}_{i \in I}$ in $\left(\mathbf{R}^{S}, l_{\infty}\right)$ has pairwise intersection at $T_{\mu}$. Each ball $B\left(p_{i}, r_{i}\right) \subseteq \mathbf{R}^{S}$ is a direct product of segments $\left[p_{i}(s)-r_{i}, p_{i}(s)+r_{i}\right]$ and thus $\left\{B\left(p_{i}, r_{i}\right)\right\}_{i \in I}$ has the Helly property on $\left(\mathbf{R}^{S}, l_{\infty}\right)$. Let $p^{*}$ be a unique maximal element in (nonempty intersection) $\bigcap_{i \in I} B\left(p_{i}, r_{i}\right)$. Then one can verify that $p^{*}$ belongs to $P_{\mu}$. Take a nonexpansive map $\phi$ in the previous lemma. Then $\phi\left(p^{*}\right)$ belongs to $\bigcap_{i \in I} T_{\mu} \cap B\left(p_{i}, r_{i}\right)$. 
Finally we verify that $T_{\mu, s}$ is normal. For a face $F$ of $P_{\mu}$, let $K_{F}$ be the graph on $S$ with edge set $E K_{F}=\{s t \mid p(s)+p(t)=\mu(s, t)(p \in F, s, t \in S)\}$. Namely $K_{F}$ represents the set of facets of $P_{\mu}$ active at $F$. Note that $K_{F}$ has a loop ss exactly when $F \subseteq\left\{p \in \mathbf{R}^{S} \mid p(s)=0\right\}$. Then $F$ belongs to $T_{\mu}$ if and only if $K_{F}$ has no isolated vertices. $T_{\mu, s}$ is the union of subcomplex of $\Delta$ since $T_{\mu, s}$ is the union of faces $F$ of $T_{\mu}$ whose $K_{F}$ has a loop at $s$. The connectivity is obvious. We show that every face containing $e$ belongs to $T_{\mu, s}$, which implies (2.4) (i). A leg coincides with an $l_{1}$-edge in the sense of [11]. Let $F$ be a minimal face of $T_{\mu}$ containing $e$. In the graph $K_{F}, s$ belongs to the connected component each of whose vertices has a loop [11, Lemma 3.8]. So every face $F^{\prime}$ of $T_{\mu}$ containing $F$ necessarily belongs to $T_{\mu, s}$. Indeed, by $E K_{F^{\prime}} \subseteq E K_{F}$, $s s \notin E K_{F^{\prime}}$ implies that $s$ is isolated in $K_{F^{\prime}}\left(F^{\prime} \nsubseteq T_{\mu}\right)$. Suppose to the contrary that $T_{\mu, s}$ violates the local convexity condition, i.e., there are two triangles $\sigma, \sigma^{\prime} \in \Delta$ of vertices $p, r, v$ and $q, r, v$ (resp.) such that $p r$ and $r q$ are hypotenuse with $T_{\mu, s} \cap\left(\sigma \cup \sigma^{\prime}\right)=p r \cap r q$. Then $\|p-q\|_{\infty}=2 \kappa$. Consider a geodesic $P$ connecting $p$ and $r$ in $\left\{p \in P_{\mu} \mid p(s)=0\right\}$. Consider the image $\phi(P)$ of $P$ by a nonexpansive map $\phi$ in Lemma 4.6. Then $\phi(P)$ is also a geodesic connecting $p$ and $q$ in $T_{\mu, s}$ with length $2 \kappa$. By (3.6) and (2.4) (i), $\Delta$ must have a triangle of vertices $p, q, v$ and hypotenuse $p q$, which violates the flag condition at $v$. A contradiction.

Remark 4.7. A metric space $X$ is called injective (or an absolute retract) if for every metric space $Y$ containing $X$ as a subspace there exists a nonexpansive retraction from $Y$ to $X$. Aronszajn and Panitchpakdi [1] showed that $X$ is injective if and only if $X$ is hyperconvex. So two Lemmas 4.5 and 4.6 are essentially equivalent.

\section{Acknowledgement}

This work is supported by a Grant-in-Aid for Scientific Research from the Ministry of Education, Culture, Sports, Science and Technology of Japan.

\section{References}

[1] N. Aronszajn and P. Panitchpakdi, Extensions of uniformly continuous transformations and hyperconvex metric spaces, Pacific Journal of Mathematics 6 (1956), 405-439.

[2] H.-J. Bandelt, Hereditary modular graphs, Combinatorica 8 (1988), 149-157.

[3] V. Chepoi, Graphs of some CAT(0) complexes, Advances in Applied Mathematics 24 (2000), 125-179.

[4] B. V. Cherkassky, Solution of a problem of multiproduct flows in a network, Èkonomika i Matematicheskie Metody 13 (1977), 143-151, (in Russian).

[5] P. M. Dearing, R. L. Francis, and T. J. Lowe, Convex location problems on tree networks. Operations Research 24 (1976), 628-642.

[6] A. W. M. Dress, Trees, tight extensions of metric spaces, and the cohomological dimension of certain groups: a note on combinatorial properties of metric spaces, Advances in Mathematics 53 (1984), 321-402.

[7] A. W. M. Dress and R. Scharlau, Gated sets in metric spaces, Aequationes Mathematicae 34 (1987), 112-120. 
[8] L. R. Ford and D. R. Fulkerson, Flows in networks, Princeton University Press, Princeton, 1962.

[9] A. Frank, A. V. Karzanov, and A. Sebö, On integer multiflow maximization, SIAM Journal on Discrete Mathematics 10 (1997), 158-170.

[10] H. Hirai, Characterization of the distance between subtrees of a tree by the associated tight span, Annals of Combinatorics 10 (2006), 111-128.

[11] H. Hirai, Tight spans of distances and the dual fractionality of undirected multiflow problems, Journal of Combinatorial Theory, Series B, to appear.

[12] H. Hirai, Bounded fractionality of the multiflow feasibility problem for demand graph $K_{3}+K_{3}$ and related maximization problems, RIMS-preprint 1645, (2008).

[13] H. Hirai, The maximum multiflow problems with bounded fractionality, in preparation.

[14] T. C. Hu, Multi-commodity network flows, Operations Research 11 (1963), 344-360.

[15] J. R. Isbell, Six theorems about injective metric spaces, Commentarii Mathematici Helvetici 39 (1964), 65-76.

[16] A. V. Karzanov, Polyhedra related to undirected multicommodity flows, Linear Algebra and its Applications 114/115 (1989), 293-328.

[17] A. V. Karzanov, Minimum 0-extensions of graph metrics, European Journal of Combinatorics 19 (1998), 71-101.

[18] A. V. Karzanov, Metrics with finite sets of primitive extensions, Annals of Combinatorics 2 (1998), 211-241.

[19] A. V. Karzanov, One more well-solved case of the multifacility location problem, Discrete Optimization, 1 (2004), 51-66.

[20] A. V. Karzanov and M. V. Lomonosov, Systems of flows in undirected networks, in: Mathematical Programming (O.I. Larichev, ed.), Institute for System Studies, Moscow, 1978, 59-66 (in Russian).

[21] A. W. J. Kolen, Tree network and planar rectilinear location theory. CWI Tract, 25, Amsterdam, 1986.

[22] M. V. Lomonosov, Combinatorial approaches to multiflow problems, Discrete Applied Mathematics 11 (1985), 93 pp.

[23] L. Lovász, On some connectivity properties of Eulerian graphs, Acta Mathematica Academiae Scientiarum Hungaricae 28 (1976), 129-138.

[24] B. C. Tansel, R. L. Francis, and T. J. Lowe, Location on networks: a survey. II. Exploiting tree network structure, Management Science 29 (1983), 498-511. 